Revue d'archéologie préhistorique

\title{
Armatures et pièces à dos du Magdalénien supérieur de La Madeleine (Tursac, Dordogne), nouvelles données de la technologie lithique
}

Alexis Taylor

\section{OpenEdition}

Journals

Édition électronique

URL : http://journals.openedition.org/paleo/2402

DOI : $10.4000 /$ paleo.2402

ISSN : 2101-0420

Éditeur

SAMRA

Édition imprimée

Date de publication : 15 décembre 2012

Pagination : 277-312

ISSN : $1145-3370$

\section{Référence électronique}

Alexis Taylor, « Armatures et pièces à dos du Magdalénien supérieur de La Madeleine (Tursac, Dordogne), nouvelles données de la technologie lithique », PALEO [En ligne], 23 | 2012, mis en ligne le 26 mars 2013, consulté le 21 décembre 2020. URL : http://journals.openedition.org/paleo/2402 ; DOI : https://doi.org/10.4000/paleo.2402

Ce document a été généré automatiquement le 21 décembre 2020.

\section{cc) (†)}

PALEO est mis à disposition selon les termes de la licence Creative Commons Attribution - Pas d'Utilisation Commerciale - Pas de Modification 4.0 International. 


\title{
Armatures et pièces à dos du Magdalénien supérieur de La Madeleine (Tursac, Dordogne), nouvelles données de la technologie lithique
}

\author{
Alexis Taylor
}

\section{Introduction}

1 Dans le cadre de cet article, nous souhaitons présenter une partie des données morphologiques et technologiques relevées sur les ensembles de pointes lithiques et lamelles à dos provenant des fouilles menées par D. Peyrony et J.-M. Bouvier à La Madeleine (commune de Tursac, Dordogne, fig. 1).

2 Situé sur la rive droite de la Vézère, s'ouvrant en pied de falaise, ce gisement d'une étendue considérable a retenu l'attention de plusieurs fouilleurs tout au long de la seconde moitié du 19è siècle. La richesse du mobilier lithique et osseux et la qualité des témoignages artistiques retrouvés consacreront La Madeleine comme site éponyme de la culture magdalénienne (Lartet et Christy 1875). Ce n'est pourtant qu'après les fouilles de D. Peyrony, entreprises en 1910 pour le compte de l'État, interrompues en octobre 1913 (la fouille concernant le centre et la partie ouest du gisement), puis reprises en 1926 (à l'est), qu'une stratigraphie de la cavité est publiée (Capitan et Peyrony 1928). Le fouilleur distingue alors trois ensembles archéologiques au sein des occupations magdaléniennes, les couches inférieure, moyenne et supérieure (ou A, B et C) (fig. 2). La composition de leurs industries lui permet alors de confirmer la division tripartite du Magdalénien proposée par l'abbé Breuil (Breuil 1912). Il faut attendre la reprise des fouilles à La Madeleine par J.-M. Bouvier en 1968 pour que la lecture de la stratigraphie de D. Peyrony évolue, avec la reprise du témoin ouest puis l'extension latérale des 
travaux en direction du centre de l'abri (Bouvier 1973, 1982). Le découpage de la séquence de remplissage est affiné, les grands horizons culturels A, B et C étant subdivisés en plusieurs occupations distinctes. Le fouilleur découvre également sous la couche A des niveaux non atteints par D. Peyrony, sous les sables d'inondations que ce dernier prenait pour le sol naturel du gisement. La nouvelle stratigraphie proposée conserve les trois ensembles sédimentaires isolés par D. Peyrony mais subdivise alors le remplissage d'une douzaine de mètres de haut en 19 niveaux géologiques et 18 niveaux d'occupations individualisés (fig. 3). A la couche inférieure correspondent alors les niveaux 30 à 26 , à la couche moyenne les niveaux 25 à 20, et à l'ensemble supérieur, les niveaux 19 à 14 .

3 Après une première approche globale des comportements techniques mis en œuvre à La Madeleine (Bundgen 2002), l'étude technologique des pièces à dos recueillies par D. Peyrony et J.-M. Bouvier, et conservées au Musée National de Préhistoire des Eyzies-deTayac, nous permet d'explorer la diversité de ces objets et de proposer les premiers éléments pour leur évolution. Des pistes interprétatives, développées dans le cadre d'une réflexion plus large sur la conception de l'armement de chasse magdalénien, notamment à travers l'examen des stratégies mises en œuvre lors de la fabrication de pointes de projectile composites (Taylor 2009), sont proposées en conclusion. Ces dernières permettent de proposer des hypothèses quant à la diversité des lamelles à dos sur le gisement de La Madeleine. Seules les données relevées sur quelques-uns des niveaux principaux seront exposées : couches A, B, C des séries Peyrony ; niveaux 27, 25 et 19 des séries Bouvier, occupations respectivement attribuées aux Magdalénien IV, $\mathrm{V}$ et VI (tab.1). Avant d'aborder la présentation de ces données, il convient de préciser l'objet de notre étude et notamment ce qu'on entend par pièces à dos.

\section{1 - Les pièces à dos : une définition morphologique et technique d'une catégorie large d'objets qui varie dans le temps et l'espace}

Il s'agit d'un ensemble d'objets partageant des méthodes et des modalités de retouche commune : un bord du support est abattu par retouche abrupte ou semi- abrupte, formant alors un dos. Des lamelles à dos, des lames à bord abattu, des pointes à dos ont donc été décrites au sein de divers assemblages lithiques du Paléolithique supérieur. La définition de "pièces à dos » devient alors double, à la fois morphologique, puisqu'on peut distinguer la nature du support ou bien la silhouette de l'objet (une pointe par exemple), et technique avec l'emploi de la retouche abrupte ou semi-abrupte que suppose le qualificatif « à dos ». 
Figure 1 - Localisation géographique du gisement de La Madeleine.

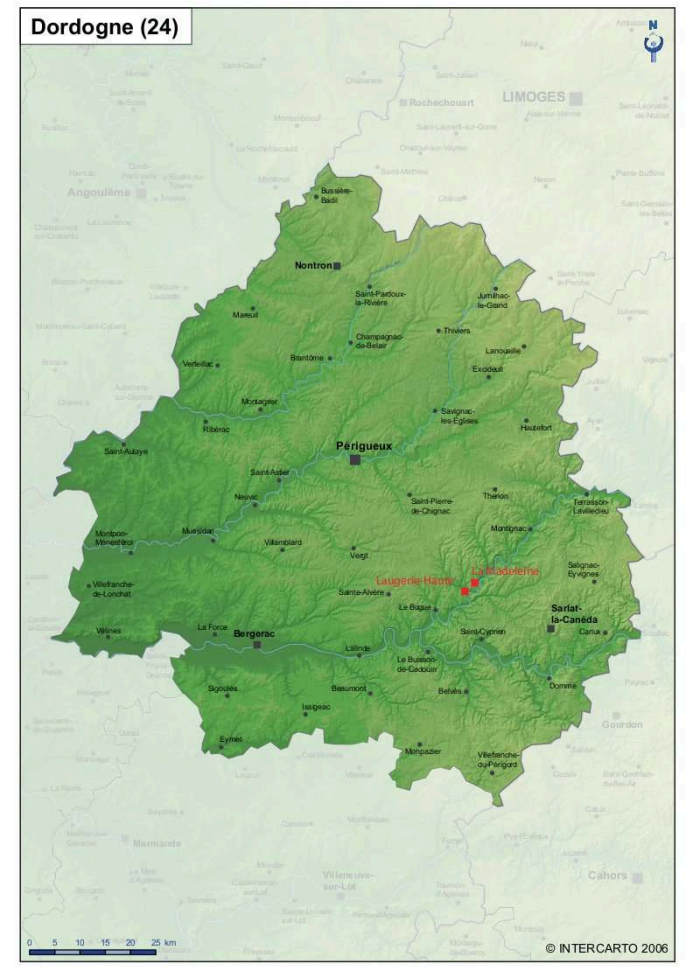

5 Parmi ces objets, les lamelles à dos sont systématiquement présentes dans les ensembles lithiques du Magdalénien moyen et supérieur, le plus souvent en abondance. Par leur présence numérique, ces objets sont ceux qui se prêtent le mieux à l'analyse de leurs modalités de fabrication, à l'observation de variations, voire d'une éventuelle évolution. Des études strictement typologiques, et plus récemment technologiques consacrées aux caractères des lamelles à dos magdaléniennes, on peut retenir une certaine variété morphologique. Fabriquées le plus souvent sur lamelle, mais aussi sur petite lame, les « lamelles à dos » au sens large, recouvrent en fait une variété de types définis en fonction des différents modes de retouche de leur support (SonnevilleBordes et Perrot 1956; Tixier 1963 ; Brézillon 1968). Les listes typologiques établies pour les industries du Paléolithique supérieur, en grande partie sur des observations faites sur des ensembles lithiques du sud-ouest de la France, comptent plusieurs grands types de lamelles à dos («simple", tronquée, denticulée par exemple), subdivisés ensuite selon les caractères de leur retouche (délinéation, orientation, régularité...). Leurs taux peuvent varier (Sonneville-Bordes 1960), mais les lamelles à dos simples (sans retouche complémentaire des extrémités ou du bord opposé au dos de l'objet) dominent systématiquement les autres classes, plus rares. Bien que partageant un trait technique commun, l'abattage d'un bord latéral du support par retouche plus ou moins abrupte, les modalités de retouche des différentes parties de ces objets sont en fait très variables selon que l'on considère le dos lui-même, le tranchant latéral qui lui est opposé ou les extrémités. En effet, ces dernières peuvent être aménagées par troncatures diversement orientées ou appointées, le bord tranchant opposé au dos abattu pouvant être laissé brut, légèrement rectifié ou fortement retouché, jusqu'à être denticulé (Piel-Desruisseaux 1998 ; Demars et Laurent 1989). 
6 Les pointes à dos se distinguent par l'aménagement d'une ou deux extrémités acuminées (pointes ou bipointes), la retouche du dos recoupant le bord latéral opposé du support. Ces objets présentent également une diversité morphologique importante, la distinction de types s'élaborant selon les modalités de la retouche de leurs supports (localisation et délinéation d'une retouche complémentaire au dos, délinéation de ce dernier), des petites lames le plus souvent (Sonneville-Bordes et Perrot, op. cit.). D'abord définies uniquement au regard de leur morphologie particulière et d'une présomption fonctionnelle, un objet pointu étant souvent considéré comme une pointe, par définition emmanchée à l'extrémité d'une hampe, ces objets ont depuis fait l'objet d'une révision critique, notamment à travers une approche fonctionnelle. Plusieurs protocoles expérimentaux, s'appuyant sur des séances de tir de répliques de pointes à dos, associés à une analyse des dommages macroscopiques (fracturation) et microscopiques (modifications des bords des pièces, polis...), ont depuis confirmé la plausibilité d'une utilisation de ces pièces comme pointes de projectile, sans que celle-ci n'exclut cependant d'autres utilisations (voir notamment les travaux récents sur les pointes à dos courbe dites « aziliennes » cf. Plisson 2005 ; Célérier et Jacquement 2005). La retouche abrupte est une solution technique largement adoptée pour la confection des pointes à dos, mais les processus responsables de leur apparition tout comme la place qu'elles occupent au sein des ensembles lithiques sont encore flous. En effet, ces dernières côtoient dans les ensembles traditionnellement attribués au Magdalénien supérieur (Magdalénien IV, V et VI) les lamelles à dos et semblent se développer plus nettement, bien qu'elles restent toujours présentes en faible nombre dans les dernières manifestations du Magdalénien, avant de devenir pour certains types plus caractéristiques de l'Epipaléolithique (pointes à dos courbes notamment). Ces pointes se développent et s'imposent en France au terme de l'époque magdalénienne, du SudOuest au Bassin parisien, dans des contextes différents. Relevant toujours en apparence d'une dynamique d'évolution interne, la technologie de ces pointes lithiques semble avoir petit à petit trouvé sa place dans la confection de l'armement magdalénien. Pour l'instant en effet, aucune rupture n'a été clairement mise en évidence entre l'émergence de ces nouveaux comportements techniques et les traditions techniques magdaléniennes (Sonneville-Bordes 1979; Bordes et Sonneville-Bordes 1979; Célérier et Moss 1983 ; Schmider 1988; Valentin 1995, 2000, 2005; Fagnart et Coudret 2000 ; Bodu 2000 ; Pelegrin 2000 ; Ladier 2003).

7 Parallèlement aux pointes à dos stricto sensu, dans le cadre d'une réflexion plus large sur les pointes de projectiles magdaléniennes, nous avons également considéré les pointes à cran magdaléniennes, des pointes à limbe court et à cran long, dégagé par retouche abrupte, dont le «dos" s'interrompt sur un cran. Dans l'état actuel des connaissances, ces pointes se développent à la fin du Magdalénien supérieur dans le sud-ouest de la France, essentiellement en Gironde et en Dordogne (Sonneville-Bordes 1960 ; Lenoir 1975, 1989 ; Lenoir et Paquereau 1987). Même s'il ne s'agit pas de pièces à dos d'un point de vue strictement typologique, elles font partie de l'armement magdalénien et sont une spécificité du gisement. Nous attachant à étudier l'évolution de cet armement, nous n'avons pas souhaité les écarter de l'étude menée sur l'abri de La Madeleine, puisqu'elles font partie de ces nouvelles formes de projectiles faisant irruption au sein des armatures magdaléniennes plus anciennes. 
Figure 2 - Coupe stratigraphique du gisement de La Madeleine par D. Peyrony (d'après Capitan et Peyrony 1928).
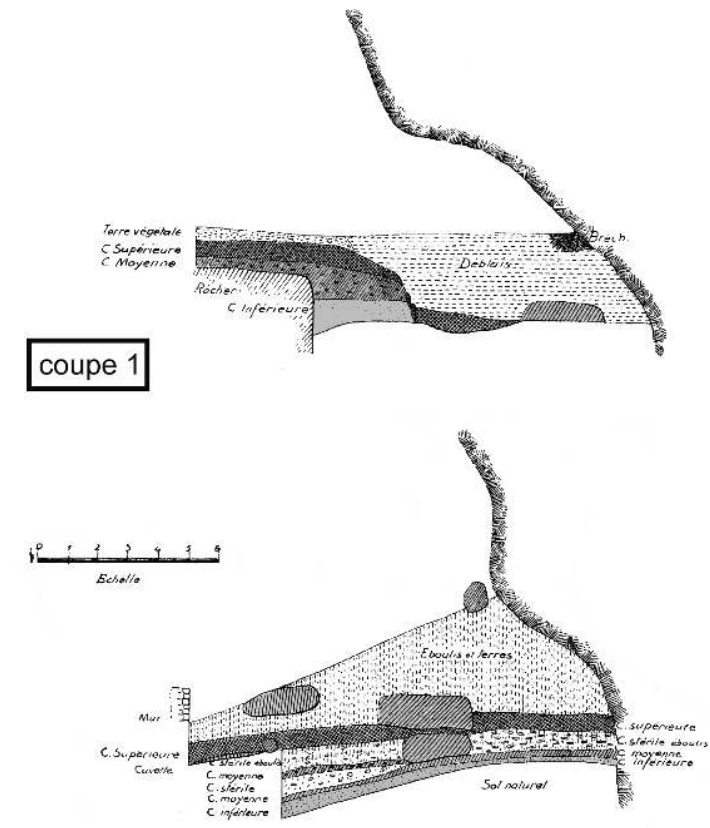

coupe 6

Figure 3 - Coupe stratigraphique du gisement de La Madeleine par J.-M. Bouvier (modifié d'après Bouvier 1973).

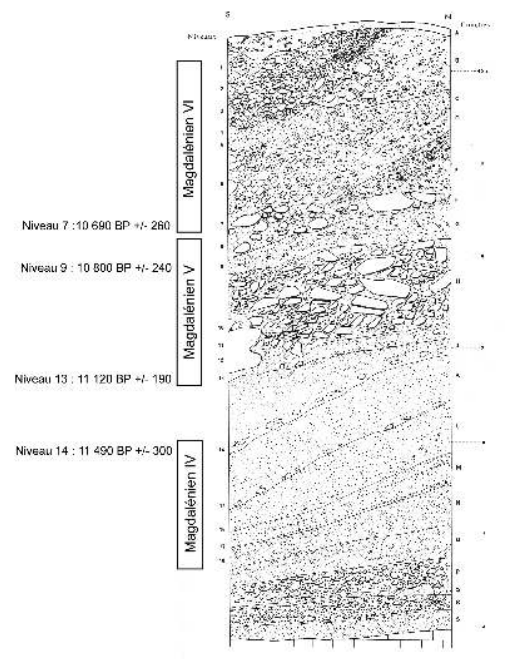


Tableau 1 - Décomptes des pièces étudiées.

\begin{tabular}{|l|c|c|c|}
\cline { 2 - 4 } \multicolumn{1}{c|}{} & pièces à dos & supports & nucléus \\
\hline Couche 27 & 337 & 398 & 59 \\
\hline Couche 25 & 299 & 281 & 31 \\
\hline couche 19 & 125 & 370 & 86 \\
\hline
\end{tabular}

\begin{tabular}{|l|c|c|c|}
\cline { 2 - 4 } \multicolumn{1}{c|}{} & pièces à dos & supports & nucléus \\
\hline Couche A & 141 & 13 & 114 \\
\hline Couche B & 206 & 10 & 94 \\
\hline Couche C & 256 & 107 & 62 \\
\hline
\end{tabular}

\section{2 - La fabrication des pièces à dos et pointes lithiques à La Madeleine}

\section{1 - Le débitage et les supports des pièces à dos}

8 D'un point de vue technologique, les méthodes de débitage restent remarquablement similaires durant l'occupation magdalénienne du gisement, nous les décrirons donc de façon synthétique. Une grande flexibilité dans les schémas de production caractérise la conception des lamelles à dos mais ces derniers obéissent néanmoins aux mêmes critères stricts quant à leur objectif.

Installés à proximité de dépôts de matière première de bonne qualité, les Magdaléniens ont principalement exploité des variétés blondes et noires de silex sénoniens locaux (Santonien, Coniacien et Campanien ; voir à ce propos notamment Turq 2005, avec une importante bibliographie) (tabl. 2). Collectés dans le lit de la Vézère coulant au pied de l'abri, sous forme de petits blocs, éclats ou plaquettes (néocortex, cortex roulés), ou bien de rognons et fragments de rognon dans les dépôts de pente (altérites) à proximité immédiate, ces matières ont permis la fabrication de la plupart des pièces à dos et pointes lithiques présentes à La Madeleine (entre 60 et $90 \%$ des objets étudiés au sein des ensembles présentés ici). Des matériaux allochtones (éloignés d'une distance comprise entre 50 et $70 \mathrm{~km}$ ) ont également été introduits et exploités de façon plus marginale. Parmi celles-ci figurent en bonne place le silex maastrichtien du Bergeracois (Séronie-Vivien et Séronie-Vivien 1987) et un silex gris blond à texture micro-grenue de type "grain de mil». Ce dernier est un silex santonien localisé dans la région de Saintes (Charentes), qui correspond au matériau dénommé "grain de mil » par R. Simonnet (Simonnet 1999 - p. 82-83 ; Simonnet et al. à paraître), mais une convergence de faciès existe avec certains blocs isolés de silex santoniens blonds provenant d'affleurements au nord du Périgord (A. Morala, communication orale).

Les tailleurs ont sélectionné des volumes de faibles dimensions (des fragments de rognons et des éclats épais) opposant une surface d'éclatement plane ou légèrement convexe à une surface naturelle plus convexe, l'arête formée par l'intersection des deux surfaces guidant les premiers enlèvements le long de la surface de débitage du nucléus. De petits rognons allongés ont également été retenus pour leurs surfaces étroites et convexes. Les surfaces de débitage sont inscrites dans l'axe longitudinal du support après une préparation rapide et plutôt simple du volume, la rapidité de la mise en forme étant autorisée à ce stade par les critères forts régissant la sélection des supports 
des nucléus. Les surfaces de débitage s'inscrivent dans un contour grossièrement triangulaire (pl. 1, 2 et 3 - en annexe), souvent présent sur les volumes sélectionnés ou ne nécessitant qu'une préparation réduite.

11 Les surfaces restent étroites, le principal schéma de débitage adoptant une progression frontale unipolaire, des enlèvements obliques plus larges en bordure de surface permettant de conserver et d'entretenir une surface étroite et triangulaire, plus fortement cintrée sur les bords (pl. 1, n 1, 3; pl. 2, n 3; pl. 3, n 1, 2, 3 - en annexe). D'autres modalités de débitage, notamment où la progression, d'abord frontale, investit ensuite progressivement un flanc du nucléus (progression frontale décalée), font intervenir l'exploitation d'un plan de frappe secondaire opposé, dont le rôle semble plutôt confiné à l'entretien de la carène et du cintre en partie inférieure du nucléus (pl. $\left.1, \mathrm{n}^{\circ} 2 ; \mathrm{pl} .2, \mathrm{n}^{\circ} 1\right)$. Ces dernières modalités ont pu être appliquées à des volumes dont l'exploitation a été plus poussée, probablement lorsque des accidents rédhibitoires pour la poursuite du débitage ont pu être évités (perte d'angulation favorable au détachement des lamelles par surcreusement de la surface de débitage ou surcreusement du plan de frappe lors de sa réfection). L'ouverture de ce plan de frappe secondaire peut intervenir dès la première phase de mise en forme mais elle semble également avoir été introduite en fonction des besoins en cours de débitage, notamment lorsque celui-ci semble plus productif. Sur certains nucléus en effet, le débitage en progressant latéralement sur un flanc, implique un pivotement latéral plus ou moins prononcé du plan de frappe secondaire, d'autant plus important que le débitage est poussé. L'organisation des enlèvements de réfection du plan de frappe principal (tablettes totales ou éclats de ravivages partiels), ainsi que la chronologie des enlèvements lamellaires sur la table (lorsqu'elle est suffisamment lisible) indiquent que cette progression latérale se fait par petites séquences juxtaposées de débitage.

12 Sur tous ces nucléus, un enlèvement plus large et oblique en bord de surface de débitage (et de fait souvent torse) permet d'obtenir la convexité et de nouvelles nervures pour revenir exploiter le volume en direction du centre de la surface de débitage où se situe la majorité des derniers enlèvements lamellaires tentés sur les volumes. En effet, les lamelles recherchées, plus étroites, sont essentiellement produites au centre de la surface de débitage où les convexités latérales et longitudinales (cintre et carène) sont entretenues par la convergence du débitage (fig. 4).

13 Il ne semble pas exister d'indépendance stricte entre les produits lamellaires et laminaires dans l'esprit des tailleurs, notamment lors de la production de supports intermédiaires, petites lames et/ou grandes lamelles. Cette indépendance est bien plus tangible dans l'économie des différents supports produits sur le gisement. Les plus petites lamelles, provenant manifestement de nucléus à vocation strictement lamellaire, sont réservées à la fabrication des lamelles à dos. Les plus grandes lamelles et petites lames, malgré la sélection de certaines d'entre elles pour la fabrication de pièces à dos (grandes lamelles à dos, pointes à dos et pointes à cran) sont plutôt transformées en outillage de fond commun tel que perçoirs, grattoirs et burins. Si d'un point de vue dimensionnel la transition entre lames et lamelles semble plutôt réalisée de façon progressive, ces produits sont néanmoins plus fortement distingués dans leur économie.

Les pièces à dos sont fabriquées sur les supports les plus réguliers, principalement des lamelles. Ces dernières sont légèrement courbes, les bords rectilignes ou légèrement 
convexes, l'extrémité distale tendant à s'effiler (pl. 4 - en annexe). Ces caractères reflètent la forte unipolarité et la convergence du débitage. Leurs dimensions varient de façon continue, mais l'observation des plus petits supports sélectionnés pour le reste de l'outillage de fond commun indique que leur présence diminue fortement dans cette catégorie de l'équipement magdalénien quand la largeur et l'épaisseur du support sont inférieures respectivement à 12 et $3 \mathrm{~mm}$. Les outils dont la longueur est inférieure à 25 $\mathrm{mm}$ sont très rares, comme on peut également le vérifier sur les lamelles à dos intactes, alors que peu de nucléus débités présentent une hauteur résiduelle inférieure à $30 \mathrm{~mm}$. Les mesures relevées sur une sélection des plus petits supports lamino-lamellaires indiquent que leur longueur varie essentiellement entre 25 et $60 \mathrm{~mm}$. Au-delà, les supports sont plutôt laminaires et tendent à être réservés à la fabrication des grandes pointes lithiques (pl. 5 et 6 - en annexe) et à l'outillage de transformation magdalénien.

Tableau 2 - Matières premières des pièces à dos.

\begin{tabular}{|c|c|c|c|c|c|c|}
\cline { 2 - 7 } \multicolumn{1}{c|}{} & \multicolumn{2}{c|}{ niveau 27 } & \multicolumn{2}{c|}{ niveau 25 } & \multicolumn{2}{c|}{ niveau 19 } \\
\hline sénonien blond & 205 & $60,8 \%$ & 96 & $32,1 \%$ & 55 & $44 \%$ \\
\hline sénonien gris & 107 & $31,8 \%$ & 84 & $28,1 \%$ & 55 & $44 \%$ \\
\hline bergeracois & 2 & $0,6 \%$ & 21 & $7 \%$ & 0 & $0 \%$ \\
\hline jaspe & 1 & $0,3 \%$ & 1 & $0,3 \%$ & 1 & $0,8 \%$ \\
\hline micro-grenu & 2 & $0,6 \%$ & 34 & $11,4 \%$ & 5 & $4 \%$ \\
\hline calcédoine & 0 & $0 \%$ & 1 & $0,3 \%$ & 3 & $2,4 \%$ \\
\hline indét/patiné & 20 & $5,9 \%$ & 62 & $20,7 \%$ & 6 & $4,8 \%$ \\
\hline total & 337 & $100 \%$ & 299 & $100 \%$ & 125 & $100 \%$ \\
\hline
\end{tabular}

\section{2 - Les dimensions des lamelles à dos}

Plusieurs gabarits semblent recherchés pour les lamelles à dos, notamment si l'on considère la largeur des objets, les variations de leur épaisseur étant beaucoup plus faibles (fig. 5 et 6). Une forte marge de variation semble en effet tolérée pour ces gabarits : les largeurs varient essentiellement entre 3 et $8 \mathrm{~mm}$, avec un pic autour de $4 \mathrm{~mm}$ pour le Magdalénien IV, autour de $5 \mathrm{~mm}$ pour le Magdalénien $\mathrm{V}$, et s'organisant de façon plus large entre 4 et $7 \mathrm{~mm}$ pour le Magdalénien VI, pour une épaisseur variant entre 1,5 et $3 \mathrm{~mm}$. Ces valeurs pourraient être considérées comme représentatives d'un gabarit "moyen» regroupant la plupart des lamelles à dos de La Madeleine, où le module de ces objets peut néanmoins varier du simple au double. Des pièces à dos peuvent dépasser les $8 \mathrm{~mm}$ de largeur. En règle générale plus robustes, ces dernières présentent une largeur après retouche qui varie entre 9 et $15 \mathrm{~mm}$, pour une épaisseur comprise entre 2 et $5 \mathrm{~mm}$. Certaines d'entre elles sont des pointes à dos, fabriquées sur lames. D'autres peuvent néanmoins représenter un objectif supplémentaire dans la fabrication des lamelles à dos sur le gisement.

Ainsi deux objectifs pourraient avoir été recherchés par les chasseurs magdaléniens, l'objectif principal étant la production de lamelles à dos de dimensions moyennes, les lamelles à dos plus robustes représentant un objectif plus secondaire. Ces objectifs évoluent cependant dans le temps, des différences significatives apparaissant notamment lorsqu'on compare statistiquement la distribution des largeurs et les épaisseurs des objets (test de Kolmogorov-Smirnov positif).

Les lamelles à dos sont fabriquées sur des supports lamellaires entiers. L'hypothèse d'une segmentation volontaire du support, afin de ne conserver par exemple qu'une longueur donnée de la partie mésiale, ne s'accorde pas avec l'analyse de la 
fragmentation des lamelles. On constate en effet que les longueurs des fragments de lamelles à dos et des supports lamellaires bruts sont comparables (test de KolmogorovSmirnov sur la distribution des fragments mésiaux à cassures simples et à fractures complexes négatif). Au contraire, les longueurs varient considérablement, avec la retouche de supports courts (moins de $30 \mathrm{~mm}$ ) correspondant aux dernières phases de débitage des nucléus, et de lamelles plus longues (mesurant entre 30 et $60 \mathrm{~mm}$, rarement plus) (fig. 7).

Figure 4 - La Madeleine : schémas d'exploitation des volumes à vocation lamellaire.

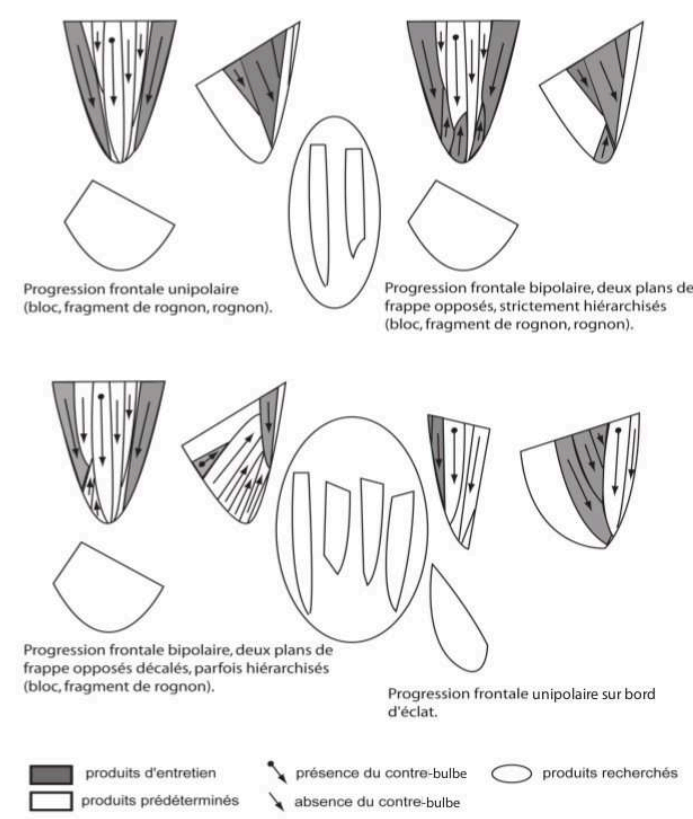

\section{3 - La retouche des lamelles à dos}

semi-abrupte plutôt qu'abrupte. D'intensité variable, la retouche s'amenuise souvent et s'interrompt même avant l'extrémité distale du support (contrairement aux pointes lithiques). Les lamelles à dos présentent alors une silhouette dissymétrique : le bord tranchant de l'objet s'incurvant en partie distale du support, une extrémité de la pièce est plus effilée que l'autre. Son orientation ne peut plus alors se faire de façon indifférente. La profondeur de la retouche peut également changer, variant d'une retouche très légère du bord de la lamelle ou la fabrication d'un dos mince par retouche marginale (la largeur du dos reste inférieure à la moitié de l'épaisseur maximale du support, le bord est légèrement abattu, à un dos plus épais abattu par une retouche plus envahissante (la largeur du dos excède la moitié ou correspond à l'épaisseur maximale de la lamelle, le bord est fortement abattu cf. «dos envahissant ou total », Christensen et Valentin in Pigeot 2004).

Les proportions entre dos fins et dos plus épais évoluent au cours de l'occupation de La Madeleine : les dos plus épais dominent durant le Magdalénien IV ( $85 \%$ de dos larges 
contre $15 \%$ de dos étroits pour le niveau 27), la présence des dos épais et plus fins s'équilibrant plus au cours des Magdaléniens V et VI (41\% de dos larges contre $59 \%$ pour le niveau $25 ; 62 \%$ de dos larges contre $38 \%$ pour le niveau 19). Le recours à une retouche plus ou moins forte n'apparaît pas forcément lié aux dimensions du support choisi, la fabrication d'un dos épais n'étant pas réservée aux lamelles les plus larges et épaisses. Il semble plus vraisemblablement lié à la volonté d'obtenir une largeur donnée pour la lamelle à dos à partir d'une production lamellaire de dimensions variées. Les populations de lamelles à dos minces et plus épais se recouvrent (fig. 8 et 9). La plupart de ces objets, qui pourrait être considérés comme étant de taille moyenne, présentent indifféremment un dos mince ou plus fortement abattu (test de Kolmogorov-Smirnov négatif). La production d'une large gamme de supports lamellaires permet alors de sélectionner différents supports (notamment en largeur) mais d'obtenir néanmoins des objets comparables en largeur et épaisseur en retouchant plus ou moins fortement le bord de la lamelle.

Les schémas de retouche de ces objets évoluent clairement durant l'occupation de l'abri, notamment en ce qui concerne l'utilisation et la localisation d'une retouche complémentaire du bord tranchant des lamelles à dos (tabl. 3 et 4). La fréquence de cette retouche, quel que soit le type considéré (à l'exception des lamelles à dos denticulées), apparaît en constante augmentation, dépassant dans certains ensembles lithiques du Magdalénien VI celle des lamelles à dos sans retouche complémentaire. La fréquence de ces pièces (séries Bouvier) oscille entre 7 et $10 \%$ pour le Magdalénien IV (niveaux 28 à 26), entre 19 et $28 \%$ pour le Magdalénien V (niveaux 25 et 24), jusqu'à atteindre un maximum de $41 \%$ au cours du Magdalénien VI (niveau 19). Dans les séries Peyrony, cette augmentation semble plus brutale, puisque les fréquences évoluent de 23 et $26 \%$ respectivement pour les couches $\mathrm{A}$ et $\mathrm{B}$, à $62 \%$ pour la couche $\mathrm{C}$. Cette dernière cependant demande probablement à être nuancée compte-tenu de la sélection dont semblent avoir fait l'objet ces séries (cf. Taylor 2009). Bien que l'on constate des variations par exemple dans l'intensité, l'orientation, ou l'angle de cette retouche, on peut également remarquer que différentes intentions semblent guider son emploi.

Une première intention pourrait correspondre au besoin de rectifier la délinéation du bord tranchant de la lamelle, comme cela semble être le cas pour les lamelles à dos présentant une retouche marginale localisée en partie distale du support, soulignant son caractère effilé (pl. 7 - en annexe). La retouche que l'on observe sur le limbe ou sur la soie des pointes à cran magdaléniennes pourrait également correspondre à une volonté similaire (pl. 5 et 6 - en annexe). Les lamelles à dos présentant une retouche semi-abrupte plus marginale que la retouche façonnant le dos, localisée en partie mésiale mais pouvant s'étendre à toute la longueur du tranchant, pourraient également relever de cette intention (pl. $8 \mathrm{n}^{\circ}$ 1-7, 10-14 et 16-17 - en annexe). La délinéation recherchée, rectiligne ou légèrement convexe, semble en effet davantage correspondre à une rectification de la morphologie du bord tranchant, plutôt qu'à un effort supplémentaire de calibration de ces objets. Cette retouche complémentaire peut alors être considérée comme un investissement supplémentaire mais qui, quoique très modique, parfois, indique qu'un soin particulier est accordé à la morphologie de ces lamelles à dos.

D'autres intentions se manifestent à travers une modification plutôt qu'une simple rectification $\mathrm{du}$ bord tranchant. Les encoches présentes sur les lamelles à dos denticulées par exemple, modifient fortement le caractère tranchant du bord. D'autres 
transformations, parfois plus discrètes, peuvent également correspondre à une modification. Plusieurs lamelles portent une retouche directe marginale, semi-abrupte et de délinéation convexe en partie proximale. Selon les objets, cette retouche complémentaire lui prête un caractère symétrique ou dissymétrique par rapport à son axe longitudinal (pl. 9 - en annexe). Dans certains cas, le même type de retouche aménage un petit cran en partie proximale de la lamelle (Magdalénien V et VI). Sur d'autres lamelles, la retouche complémentaire est étendue à la totalité du bord, accentuant ou façonnant véritablement un bord convexe : bien qu'elle reste régulière, la retouche devient plus profonde à proximité des extrémités du support (pl. $8 \mathrm{n}^{\circ}$ 9, 15 et 18 - en annexe) (Magdalénien VI). Sur toutes ces pièces, la retouche complémentaire dépasse la simple rectification du bord de la lamelle. Il pourrait s'agir d'aménagements spéciaux, en relation avec l'utilisation et/ou l'emmanchement de ces objets. Il s'agit là d'une question difficile à résoudre à La Madeleine où la grande variabilité des lamelles à dos (c'est aussi le cas des pointes lithiques, dont la relative rareté complique encore la perception de leur diversité) s'appréhende plus aisément à travers leurs mensurations mais beaucoup plus difficilement par l'observation de critères plus discrets, comme cette retouche complémentaire qui parfois modifie très peu la morphologie originale du support choisi. Certains caractères morphologiques (bord convexe, bord rectiligne, rétrécissement proximal, extrémité distale pointue) sont en effet présents sur les produits de débitage sans qu'une retouche complémentaire soit nécessaire. La discrétion de ces modifications pourrait nous conduire à minimiser la signification de cette retouche complémentaire, si son développement ne se faisait pas de façon progressive, sa présence permettant de distinguer des classes au sein des lamelles à dos, dont la présence parmi des assemblages lithiques évolue de façon nettement orientée dans le temps (fig. 10 et 11).

Figure 5 - Largeurs des pièces à dos, de haut en bas : couches 27, 25, 19.
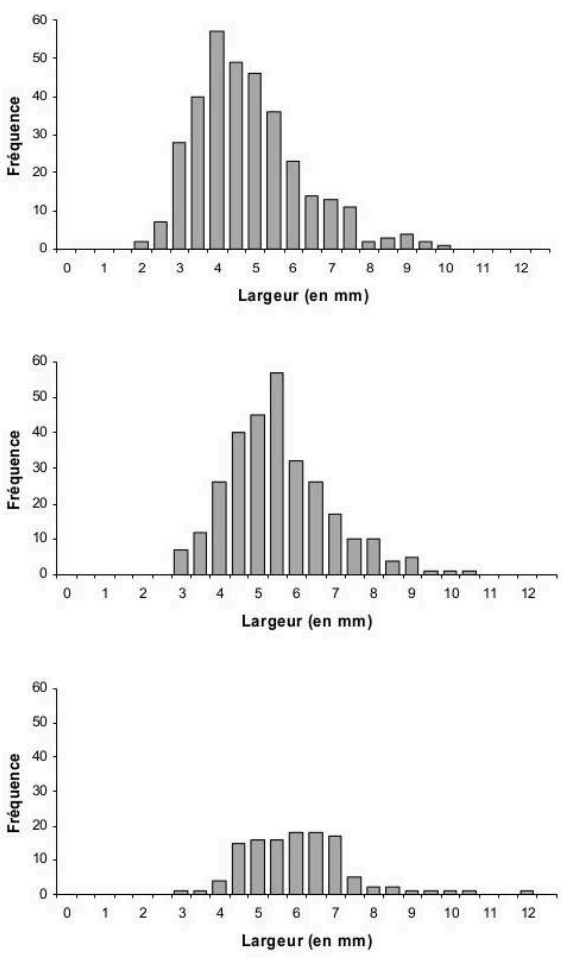
Figure 6 - Epaisseurs des pièces à dos, de haut en bas : couches 27, 25, 19.
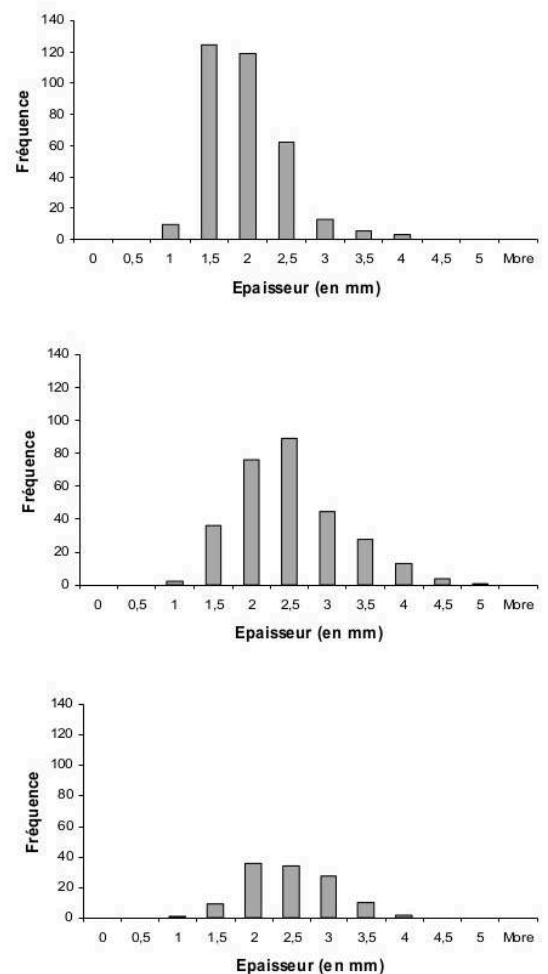

Figure 7 - Longueurs des fragments mésiaux de lamelles à dos simples, de haut en bas : couches $27,25,19$.
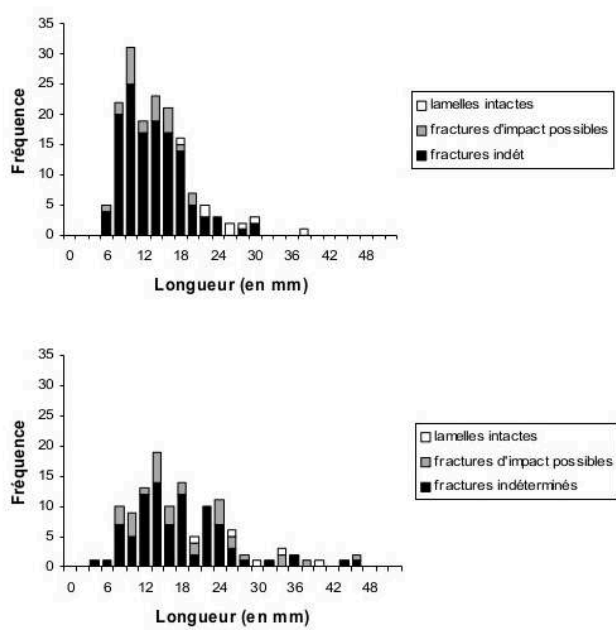

Dlamelles intactes

afractures dimpact pos sib

- fractures indéterminé

Longueur (en $\mathrm{mm}$ )

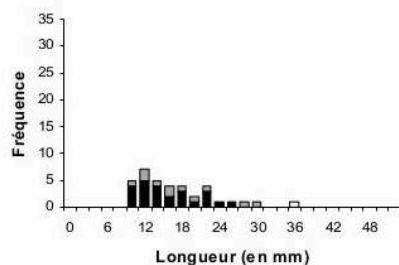

alamelles intactes 
Figure 8 - Largeurs des pièces à dos, de haut en bas : couches 27, 25, 19.
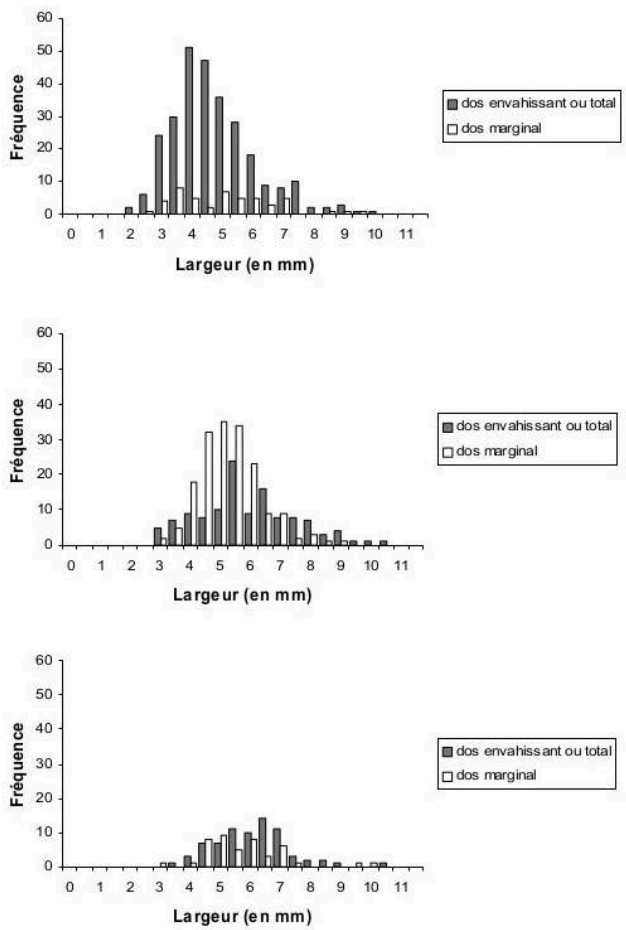

Figure 9 - Epaisseurs des pièces à dos, de haut en bas : couches 27, 25, 19.
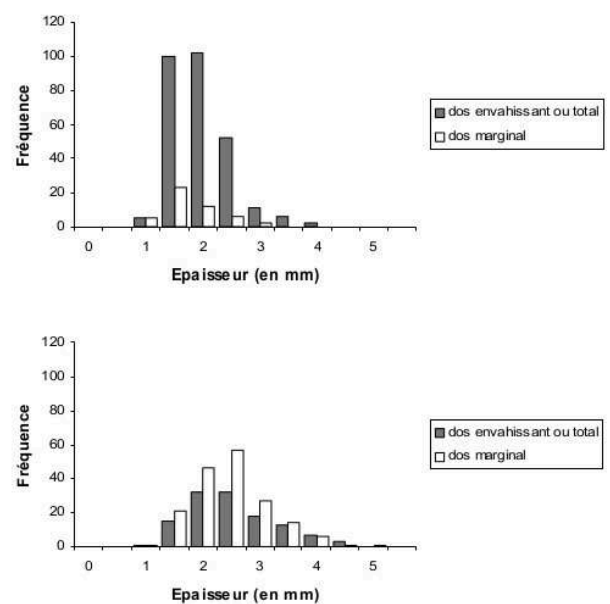

$\square$ dos marginal

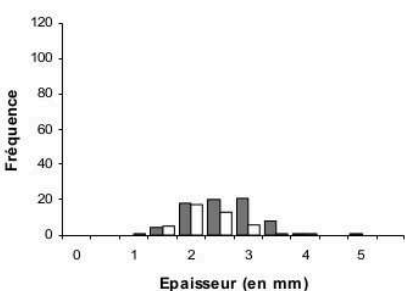

- dos envahis sant ou total

$\square$ dos marginal 
Tableau 3 -Fréquences des classes de pièces à dos. Séries D. Peyrony.

\begin{tabular}{|c|c|c|c|c|c|c|}
\cline { 2 - 7 } \multicolumn{1}{c|}{} & \multicolumn{2}{c|}{ couche A } & \multicolumn{2}{c|}{ couche B } & \multicolumn{2}{c|}{ couche C } \\
\hline classes & $\mathbf{n}$ & $\%$ & $\mathbf{n}$ & $\%$ & $\mathbf{n}$ & $\%$ \\
\hline Ids & 87 & $61,7 \%$ & 103 & $50 \%$ & 39 & $15,2 \%$ \\
\hline Idop & 15 & $10,6 \%$ & 31 & $15,1 \%$ & 20 & $7,8 \%$ \\
\hline Idod & 3 & $2,1 \%$ & 9 & $4,4 \%$ & 21 & $8,2 \%$ \\
\hline Idor & 14 & $9,9 \%$ & 15 & $7,3 \%$ & 107 & $41,8 \%$ \\
\hline Idd & 5 & $3,5 \%$ & 12 & $5,8 \%$ & 10 & $3,9 \%$ \\
\hline Ide & 5 & $3,5 \%$ & 2 & $1 \%$ & 0 & $0 \%$ \\
\hline Id ang & 0 & $0 \%$ & 0 & $0 \%$ & 0 & $0 \%$ \\
\hline Idt & 2 & $1,4 \%$ & 4 & $1,9 \%$ & 3 & $1,2 \%$ \\
\hline Iscal & 0 & $0 \%$ & 0 & $0 \%$ & 1 & $0 \%$ \\
\hline pd & 3 & $2,1 \%$ & 11 & $5,3 \%$ & 6 & $2,3 \%$ \\
\hline pdc & 1 & $0,7 \%$ & 3 & $1,5 \%$ & 7 & $2,7 \%$ \\
\hline pcm & 0 & $0 \%$ & 3 & $1,5 \%$ & 29 & $11,3 \%$ \\
\hline pièce à cran & 2 & $1,4 \%$ & 5 & $2,4 \%$ & 8 & $3,1 \%$ \\
\hline Idco & 2 & $1,4 \%$ & 1 & $0,5 \%$ & 0 & $0 \%$ \\
\hline indét & 2 & $1,4 \%$ & 7 & $3,4 \%$ & 5 & $2 \%$ \\
\hline n total & $\mathbf{1 4 1}$ & $\mathbf{1 0 0} \%$ & $\mathbf{2 0 6}$ & $\mathbf{1 0 0} \%$ & $\mathbf{2 5 6}$ & $\mathbf{1 0 0} \%$ \\
\hline
\end{tabular}

Tableau 4 - Fréquences des classes de pièces à dos. Séries J.-M. Bouvier.

\begin{tabular}{|c|c|c|c|c|c|c|}
\cline { 2 - 7 } \multicolumn{1}{c|}{} & \multicolumn{2}{c|}{ niveau 27 } & \multicolumn{2}{c|}{ niveau 25 } & \multicolumn{2}{c|}{ niveau 19 } \\
\hline classes & $\mathbf{n}$ & $\%$ & $\mathbf{n}$ & $\%$ & $\mathbf{n}$ & $\%$ \\
\hline Ids & 277 & $82,2 \%$ & 209 & $69,9 \%$ & 59 & $47,2 \%$ \\
\hline Idop & 13 & $3,9 \%$ & 27 & $9 \%$ & 17 & $13,6 \%$ \\
\hline Idod & 11 & $3,3 \%$ & 17 & $5,7 \%$ & 3 & $2,4 \%$ \\
\hline Idor & 11 & $3,3 \%$ & 13 & $4,4 \%$ & 31 & $24,8 \%$ \\
\hline Idd & 2 & $0,6 \%$ & 20 & $6,7 \%$ & 1 & $0,8 \%$ \\
\hline Ide & 2 & $0,6 \%$ & 2 & $0,7 \%$ & 0 & $0 \%$ \\
\hline Id ang & 0 & $0 \%$ & 0 & $0 \%$ & 1 & $0,8 \%$ \\
\hline Idt & 8 & $2,4 \%$ & 0 & $0 \%$ & 3 & $2,4 \%$ \\
\hline Iscal & 4 & $1,2 \%$ & 0 & $0 \%$ & 0 & $0 \%$ \\
\hline pd & 3 & $0,9 \%$ & 1 & $0,3 \%$ & 0 & $0 \%$ \\
\hline pdc & 2 & $0,6 \%$ & 2 & $0,7 \%$ & 0 & $0 \%$ \\
\hline pcm & 0 & $0 \%$ & 0 & $0 \%$ & 1 & $0,8 \%$ \\
\hline pièce à cran & 4 & $1,2 \%$ & 5 & $1,7 \%$ & 6 & $4,8 \%$ \\
\hline Idco & 0 & $0 \%$ & 0 & $0 \%$ & 0 & $0 \%$ \\
\hline indét & 0 & $0 \%$ & 3 & $1 \%$ & 3 & $2,4 \%$ \\
\hline n total & $\mathbf{3 3 7}$ & $\mathbf{1 0 0} \%$ & $\mathbf{2 9 9}$ & $\mathbf{1 0 0} \%$ & $\mathbf{1 2 5}$ & $\mathbf{1 0 0} \%$ \\
\hline
\end{tabular}

Légende tab. 3 et 4 - Ids : lamelle à dos simple ; Idop : lamelle à dos à retouche opposée proximale : Idod : à retouche opposée distale ; Idor : à retouche du bord opposé ; Idd : lamelle à dos denticulée : Ide : lamelle à dos avec encoche sur le bord opposé ; Id ang : lamelle à dos anguleux; Idt : lamelle à dos tronquée ; Iscal : lamelle scalène ; pd : pointe à dos arqué ; pdc : pointe à dos courbe ; pcm : pointe à cran magdalénienne; Idco : lamelle à dos avec cran sur le bord opposé ; indét : pièce avec retouche abrupte indéterminée 


\section{3 - Des pièces à dos aux armatures lithiques}

\section{1 - L'utilisation des pièces à dos}

23 Plusieurs pièces à dos (lamelles et pointes lithiques) présentent des ébréchures et fractures complexes (Fischer et al. 1984) que l'on peut considérer comme diagnostiques d'une utilisation comme éléments de projectile (Célérier et Moss 1983; Plisson et Geneste 1989; Geneste et Plisson 1990, 1993 ; Chadelle et al. 1991 ; Cattelain et Perpère 1993, 1996; Soriano 1998; O'Farrell 2004 ; O'Farrell et Pelegrin 2005 ; Plisson 2005). Il s'agit de fractures en flexion particulièrement développées (languettes avec une terminaison en marche parfois incipiente, charnières transversales), d'enlèvements pseudo-burinants emportant une partie du bord opposé ou du dos de l'objet (parfois sur une longueur considérable, dépassant le centimètre), pour les dommages les plus importants. Des modifications plus discrètes du bord tranchant des pièces sont également visibles, ce sont de petits enlèvements envahissants rasants, obliques par rapport à l'axe d'allongement de la pièce, ou des arrachements plus importants sur le bord actif (pl. 10, 11 et 12 - en annexe). Les pointes lithiques et les lamelles à dos partagent ces stigmates (toutes classes d'objets lithiques et gabarits confondus), les ébréchures étant cependant plus fréquentes sur le bord des lamelles à dos, l'amplitude des fractures étant dans l'ensemble plus faible également sur ces pièces, à quelques exceptions près. Ces observations peuvent être un indice, bien que ténu, d'un emmanchement plutôt latéral de ces pièces, exposant moins leurs extrémités aux chocs.

L'emmanchement latéral de lamelles à dos sur une pointe de projectile en matière dure d'origine animale, sans exclure une origine végétale du support, est une hypothèse largement acceptée depuis la découverte exceptionnelle d'un fragment mésial de pointe de sagaie en bois de cervidé encore serti de lamelles de silex à Pincevent (Seineet-Marne), et de lamelles sur lesquelles adhérait encore un mastic de fixation à Lascaux (Dordogne) (Leroi-Gourhan 1983; Allain et Leroi-Gourhan 1979). Malheureusement, si des expériences de tir de projectile ont essentiellement concerné l'utilisation de traits armés d'une pointe lithique, des stigmates propres à l'utilisation d'une armature latérale, fonction qu'on attribue volontiers aux lamelles à dos, n'ont pas encore été aussi bien définis. Une fonction exclusive d'armature a pu parfois être remise en cause pour les lamelles à dos, sans que l'on puisse préciser si cette autre utilisation (découpe) avait été mise en œuvre dans le cadre d'une utilisation occasionnelle liée au caractère pratique de l'élément pour la tâche envisagée, ou d'un réemploi (Keeley In Audouze 1981 ; Moss et Newcomer 1982). Si les stigmates décrits plus haut peuvent être considérés comme caractéristiques d'une utilisation de ces objets comme éléments de projectile, il apparaît difficile à La Madeleine de les distinguer des pièces ne présentant que des cassures en flexion non diagnostiques (cassures simples). Des cassures simples peuvent se produire également lors d'un impact mais ne se distinguent pas suffisamment de cassures accidentelles. Elles représentent toujours le type de cassure le plus fréquent, y compris dans des séries expérimentales dont le but avoué est de produire des fractures diagnostiques. Une autre fonction demeure possible pour ces pièces, mais leur proximité morphologique avec les objets dont l'endommagement suggère une utilisation comme éléments d'armatures ne permet pas de les distinguer 
plus avant à ce stade de l'analyse (seule une analyse tracéologique pourrait préciser ce point).

Figure 10 - Distribution des classes de pièces à dos. Série Bouvier (Analyse factorielle des correspondances : les carrés noirs représentent les classes de pièces à dos, les losanges gris les niveaux auxquels elles s'associent).

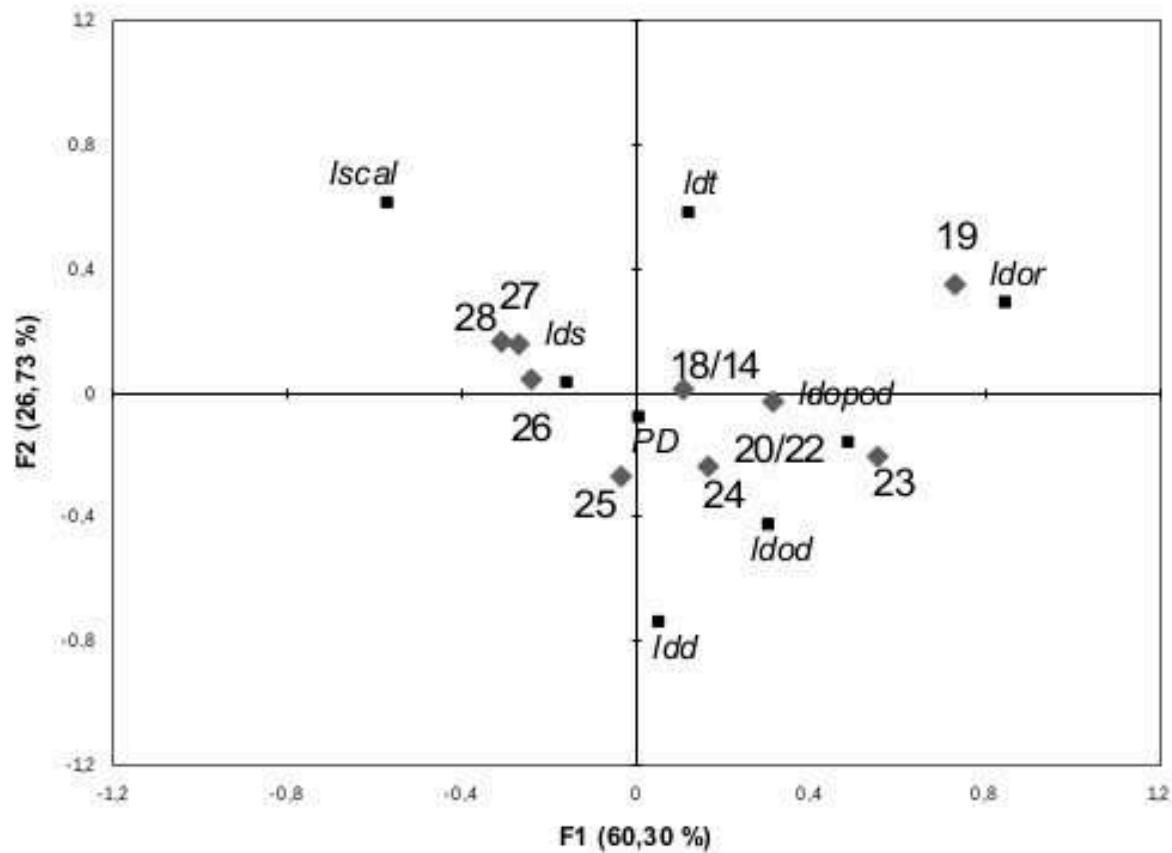

Lds : lamelle à dos simple ; Idopod : à retouche opposée proximale ; Idod : à retouche opposée distale seulement;

Idor : à retouche du bord opposé ; Idt : lamelle à dos tronquée

Idd : lamelle à dos denticulée

Iscal : lamelle scalène ; PD : pointe à dos. 
Figure 11 - Distribution des classes de pièces à dos. Série Peyrony (Analyse factorielle des correspondances : les carrés noirs représentent les classes de pièces à dos, les losanges gris les niveaux auxquels elles s'associent).

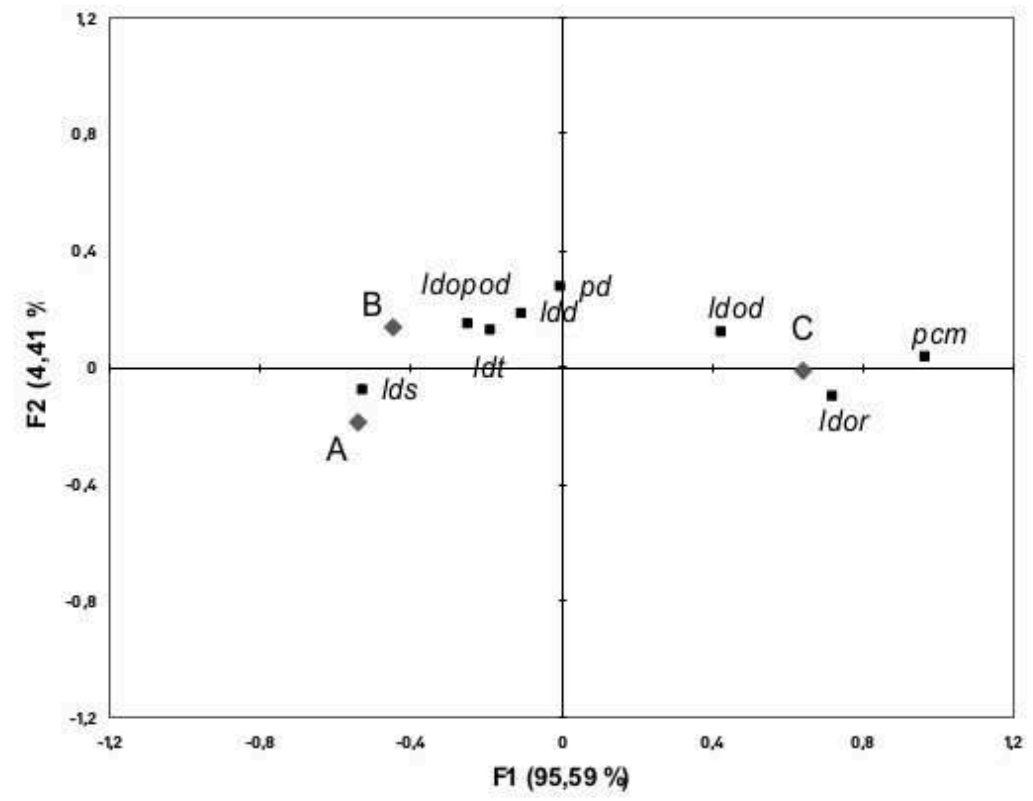

Lds : lamelle à dos simple ; Idopod : à retouche opposée proximale ; Idod : à retouche opposée distale seulement; Idor : à retouche du bord opposé ; Idt : lamelle à dos tronquée ; Idd : lamelle à dos denticulée ; Iscal : lamelle scalène ; PD : pointe à dos ; pcm : pointe à cran magdalénienne.

\section{2 - Inserts lithiques et pointes composites}

Le statut exact des lamelles à dos portant une retouche complémentaire demeure problématique. La rectification du bord tranchant peut être un argument en faveur de l'insertion en série de tels objets, la calibration des inserts et la délinéation du tranchant obtenu étant alors des facteurs importants pour l'utilisation du projectile (pénétration et entretien de la pointe). La modification du bord tranchant pourrait en revanche être l'expression d'un traitement plus individuel de ces pièces à dos et/ou une pluralité de solutions d'emmanchement : barbelures latérales uniques, présence d'une ligature (convexité ou épaulement/cran proximal), emmanchement latéro-axial voire axial pour les objets appointés ? La fragilité de ces pièces, dont la largeur et l'épaisseur sont toujours bien plus réduites que les objets définis typologiquement comme des pointes lithiques, semble problématique, à moins d'envisager une plus grande légèreté de l'ensemble du projectile et de la hampe notamment. Bien plus que la pointe, c'est cette dernière qui apportera sa masse au projectile et modifiera ses propriétés balistiques. Du point de vue de la fracturation cependant (localisation, amplitude des fractures complexes), il semble difficile pour l'instant de distinguer ces pièces du reste des lamelles à dos.

La retouche des pointes lithiques, leur morphologie et leur plus grande robustesse évoquent plutôt un emmanchement axial que latéral. Plusieurs observations à La Madeleine semblent pouvoir soutenir cette hypothèse (pl. $9, \mathrm{n}^{\circ} 6-$ en annexe). Ces objets pourraient alors être l'indice de nouvelles conceptions de pointes de projectile, 
leurs procédés de fabrication s'intégrant progressivement entre la production des lamelles à dos et le reste de l'outillage magdalénien.

La grande diversité des lamelles à dos (dimensions) nous permet en outre d'envisager une pluralité de combinaisons possibles entre une pointe de projectile organique et des inserts lithiques, soulignant la grande souplesse adaptative d'une telle association (fig. 12).

Figure 12 - La Madeleine : différentes combinaisons pour les pointes de projectiles composites.

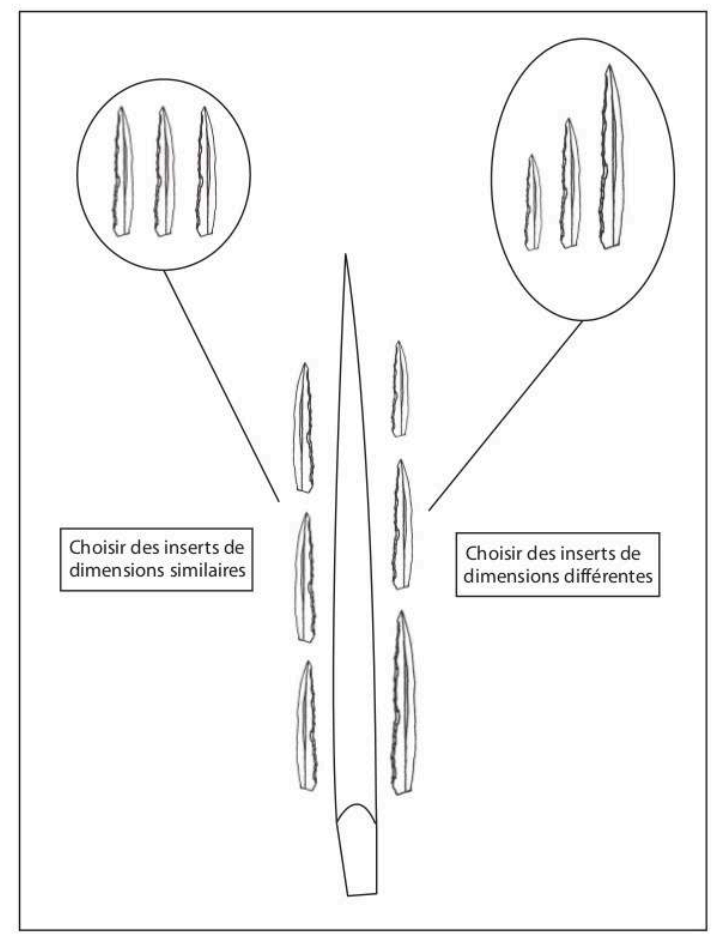

\section{4 - Premiers éléments pour une évolution des pièces à dos à La Madeleine : les indices d'une modification globale de l'équipement de chasse magdalénien?}

Le Magdalénien supérieur/final apparaît alors comme une période d'innovation et de diversification importante de l'armement de chasse à La Madeleine, avec l'introduction d'éléments barbelés et le développement de pointes lithiques. On comprend cependant mal pourquoi une telle diversité de projectiles se développe, alors que la chasse ne semble plus guère ne concerner que le Renne, et plus rarement le Cheval sur ce gisement.

Est-ce parce que toute la production d'armatures n'était pas destinée à être utilisée lors des chasses réalisées sur le gisement, mais qu'une partie des projectiles pouvait être utilisée ailleurs puis rapportée, entretenue et finalement abandonnée à La Madeleine ? Les données concernant le traitement des carcasses animales nous manquent pour apprécier l'introduction du produit de chasses menées à proximité immédiate du site ou d'expéditions plus distantes, la question reste donc ouverte. L'hypothèse d'une plus 
grande permanence de l'occupation humaine semble être soutenue à La Madeleine par les données de saisonnalité disponibles pour le Renne, mais elle n'implique pas la sédentarité de la totalité des groupes humains. Si une partie du groupe pouvait s'éloigner pour chasser avec un équipement différent, il semblerait que ce soient les mêmes gibiers qui aient été chassés.

L'abandon de harpons, voire leur fabrication à La Madeleine - quelques objets portent des traces de réfection, les supports-baguettes extraits sur place peuvent convenir à leur fabrication - pose la question de leur destination. Une chose est sûre : ils ne remplacent pas les projectiles précédents. La place exacte de l'acquisition du poisson (importance, saisonnalité) dans la subsistance des Magdaléniens de La Madeleine reste à ce jour inconnue, mais restreindre l'utilisation de ces armatures barbelées au seul domaine de la pêche nous semble difficile à justifier. La diversité des embases évoquent des têtes mobiles (butées dépassant du corps du fût au niveau de l'embase du projectile) mais aussi des têtes fixes (biseaux striés). Utilisés pour la chasse terrestre, leur propriété de rétention évoque néanmoins des techniques de capture différentes. Si la piste d'une relation privilégiée d'un type de projectile avec une espèce particulière semble devoir être définitivement abandonnée dans l'état des données disponibles à La Madeleine, on peut néanmoins suggérer l'hypothèse de leur utilisation dans des situations de chasse nécessitant des techniques de capture particulières, puisque les divers projectiles fabriqués au cours du Magdalénien VI possèdent des propriétés bien différentes, voire des armes différentes avec la légèreté de certaines armatures (propulseur/arc).

31 L'évolution des schémas de retouche des lamelles à dos est nettement plus marquée durant les Magdaléniens V et VI, quand des pointes à dos courbe tout d'abord puis des pointes à cran sont fabriquées, et que la technologie du harpon se développe dans le travail du bois de renne sur le gisement. L'évolution constatée dans la retouche des lamelles à dos pourrait-elle être interprétée comme le résultat d'un changement de statut des conceptions préexistantes de pointes de projectile, qui ne représenteraient plus les seules options techniques? Le soin supplémentaire accordé au façonnage de la morphologie finale de certaines lamelles pourrait alors correspondre à une volonté de mieux définir le rôle de l'équipement de chasse antérieur à l'introduction des nouvelles pointes de projectile. D'autres pièces à dos pourraient être liées à l'invention de nouvelles solutions d'emmanchement, ou représenter de nouvelles associations d'inserts, voire même des pointes de projectiles plus légères (petites pointes à dos ?). L'évolution des lamelles à dos pourrait donc être en relation avec une modification globale de l'équipement et des techniques de chasse magdaléniennes.

32 La question de cette modification globale de l'équipement reste délicate à appréhender à La Madeleine, notamment au sein d'un cadre chronologique élaboré autour de datations aujourd'hui obsolètes, mais le croisement des données provenant de l'étude des pièces à dos et des armatures en bois de renne (Braem 2008; Taylor 2009) a pu en suggérer certains aspects discrets.

33 Outre la recherche de nouvelles propriétés de rétention que suggère le développement de la production d'armatures barbelées, cette dernière ne semble pas sans incidence sur la fabrication des armatures de sagaie en bois de renne sur le gisement. Ainsi, la recherche de supports convenant à la fabrication des harpons, notamment double, semble avoir conduit les Magdaléniens à sélectionner des modules de bois de renne de plus en plus conséquents, autant que l'on puisse en juger d'après l'épaisseur minimale 
de compacta relevée sur les objets façonnés et baguettes-supports laissées brutes (Taylor 2009, d'après les données brutes de L. Braem). Les largeurs et épaisseurs des armatures de sagaies augmentent sensiblement au cours des Magdaléniens V et VI de La Madeleine, esquissant une évolution similaire à celle des dimensions générales des lamelles à dos et du débitage lamellaire. De même, les stratégies de fabrication de ces dernières semblent s'organiser très souplement autour d'un objectif dimensionnel moyen, constituant ainsi une gamme de pointes de projectile. L'association entre une partie au moins des lamelles à dos et des armatures de sagaie, suggérée par les dommages survenus aux inserts lithiques, semble ainsi encore renforcée par cette évolution parallèle.

34 Ces changements discrets dans l'industrie lithique lamellaire ne doivent cependant pas faire oublier la remarquable continuité des schémas de débitage lamellaire à La Madeleine dont nous avons pu souligner la remarquable persistance des principes forts et la recherche persistante d'une morphologie particulière des lamelles à dos. C'est cette continuité et le caractère très progressif des changements observés qui rendent très délicate l'observation et l'estimation de l'impact de l'introduction des pointes lithiques au sein des assemblages lithiques. En faible nombre, d'une très grande diversité morphologique et dimensionnelle, les pointes à dos semblent s'intégrer dans les productions lithiques du gisement sans rupture, façonnées sur des produits intermédiaires, à la charnière des débitages laminaire et lamellaire. Cette situation n'est pas propre à La Madeleine, puisque la filiation semble encore forte entre les productions lithiques de la fin du Magdalénien, où apparaissent ces pointes lithiques, et les premières industries aziliennes où les pointes à dos, notamment courbes, prennent leur importance ( Pion 1990, 1997 ; Bodu 1993, 1998, 2000 ; Célérier 1998; Chollet et al. 1999 ; Valentin 2000 ; Leesch et al.2004; Valentin in Cholet et Dujardin 2005).

\section{5 - Vers une conception magdalénienne d'un équipement composite?}

Une autre grande constante, d'autant plus forte qu'elle semble également présente au sein d'autres contextes magdaléniens (Taylor 2009), concerne la souplesse et le caractère adaptatif propres aux stratégies de production et de transformation des supports des inserts lithiques, caractères que nous sommes tentés d'interpréter comme un véritable principe de précaution technique garantissant l'efficacité d'un armement composite (système fiable). En effet, la sélection d'une gamme de supports lamellaires variés, assortie à une modulation du degré d'abattage du dos des pièces réduisant plus ou moins fortement la largeur originelle du support sélectionné, sont susceptibles de traduire l'armement de pointes de projectile en bois de renne de calibres différents et peuvent alors résulter d'une stratégie d'anticipation, de gestion et d'adaptation sur le temps long de l'armement de chasse. Cette gestion reposerait alors sur la fabrication d'inserts lithiques dont les modalités de production peuvent être en partie modulées au besoin. Ces besoins peuvent couvrir : l'armement de pointes de projectile différenciées (ce serait le cas dans le niveau IV-20 de Pincevent), la réfection ou l'entretien des têtes de projectile, voire des changements complets d'armatures lithiques sur un armement importé (par exemple dans le niveau Q31 d'Etiolles). Plus qu'une anticipation de la durée d'occupation, c'est plutôt la souplesse adaptative de l'équipement de chasse (en tout cas pour les armatures lithiques) qui semble guider la fabrication des projectiles 
sur différents sites magdaléniens (Taylor 2009). L'anticipation à l'échelle du cycle et pas seulement de la prochaine occupation justifierait alors l'emploi d'un armement relativement polyvalent. Elle pourrait expliquer le caractère modulable de la production des inserts lithiques sur ces gisements. Il apparait toutefois que cette polyvalence, si elle semble bien avérée, puisse, en tant que principe de prudence, faire suffisamment partie intégrante des impératifs de fabrication magdaléniens pour s'affranchir de certains facteurs de variation telles que des différences dans les saisons d'occupation, la fréquence ou la diversité des espèces chassées (voir la comparaison entre les niveaux IV-20 et IV0 de Pincevent).

La polyvalence et une certaine anticipation des besoins futurs dans la conception de l'équipement magdalénien avaient déjà été soulignées à travers les concepts mêmes sous-tendant le débitage laminaire magdalénien, où la recherche d'un produit laminaire calibré privilégiant la longueur du support autorise alors la confection d'une large gamme d'outils pouvant être ré-affûtés à l'identique ou modifiés complètement par la suite. Identifiés dans le Magdalénien supérieur du Bassin parisien tout d'abord, ces concepts semblent pouvoir s'étendre à l'ensemble du monde magdalénien.

Cette vision synthétique de plusieurs grands ensembles lithiques magdaléniens (Taylor 2009) peut sembler par trop simpliste dans sa volonté d'en retrouver les grands caractères communs. En effet, la souplesse dans les modalités de fabrication d'un équipement de chasse conçu " pour durer » dans le temps (au moins à l'échelle du cycle annuel ?), peut être un " fil conducteur » supplémentaire permettant de fédérer des ensembles magdaléniens nettement distincts par ailleurs. Mais cette stratégie commune, révélant à notre sens un véritable principe de précaution technique, ne doit cependant pas masquer des éléments plus discrets, des « tours de main » techniques qui prêtent à plusieurs ensembles magdaléniens une identité bien particulière. La présence discrète de certaines armatures, plus caractéristiques d'autres régions, qui témoignent de relations entre différents groupes de chasseurs magdaléniens, doit également être soulignée (pointes à base fourchue, Lussac-Angles, pointes lithiques à cran).

Ces traits distinctifs caractérisent la conception des armatures dans l'esprit des chasseurs magdaléniens, notamment à travers l' «image " que ces derniers pouvaient en avoir. Cette "image » peut se traduire notamment par une rigidité des schémas de retouche (latéralisation, orientation du support), des degrés de tolérance différents sur les qualités des supports (rectification de la courbure par retouche inverse, segmentation des extrémités du support, retouche complémentaire de rectification, troncature et fabrication de rectangles) voire le soin de la retouche. La conception même de l'armature dans l'esprit des chasseurs magdaléniens nous semblent donc également un facteur à ne pas négliger lorsque l'on s'attache à étudier sa morphologie, même si ce dernier, subjectif par nature, est sans doute le plus délicat à cerner.

\section{Conclusion}

Qu'elles soient anciennes ou plus récentes, l'observation fine des séries lithiques de La Madeleine, nécessaire et attendue en raison de l'importance historique du gisement, souligne la singularité de ce dernier. Cependant, à travers les questions qu'elles posent quant à la conception d'un armement de chasse composite, elles l'inscrivent pleinement au sein d'une recherche actuelle qui grâce à l'analyse technologique, expose et explore la variabilité du monde magdalénien. La synthèse des approches 
pluri-disciplinaires menées à La Madeleine et sur d'autres grands gisements magdaléniens européens, imparfaite car toujours en construction, permet à notre sens d'en souligner plusieurs principes forts. D'autres études technologiques sont bien entendu nécessaires pour renforcer les traits d'un tableau qui s'esquisse et auquel nous espérons avoir contribué avec ce travail.

Nous tenons à remercier Monsieur Jean-Jacques Cleyet-Merle, Directeur du Musée National de Préhistoire, pour nous avoir autorisé à étudier le matériel de La Madeleine, ainsi que Madame Catherine Perlès, Professeur à l'université de Nanterre, qui a accepté de diriger nos travaux de doctorat.

Nos plus vifs remerciements vont également à André Morala, pour sa disponibilité et son amitié, ainsi qu'à Jacques Pelegrin pour ses conseils et nos échanges sur le mobilier.

Lucie Braem nous a témoigné la plus grande confiance en acceptant de nous confier une partie de ses données sur l'industrie en bois de cervidé de La Madeleine, qu'elle en soit ici remerciée.

\section{BIBLIOGRAPHIE}

ALLAIN J., LEROI-GOURHAN Arl. 1979 - Lascaux inconnu. Gallia Préhistoire, Supplément 12. Paris : CNRS, $386 \mathrm{p}$.

BODU P. 1993 - Analyse typo-technologique du matériel lithique de quelques unités du site magdalénien de Pincevent (Seine-et-Marne). Applications spatiales, économiques et sociales. Thèse de doctorat, Université de ParisI, 3 volumes, 852 pages.

BODU P. 1998 - Magdalenians-Early Azilians in the centre of the Paris basin : A filiation? The example of the Closeau (Rueil-Malmaison, France).InS. Miliken (dir.), The Organization of Lithic Technology in Late Glacial and Early Postglacial of Europe.BAR International Series, 700, Oxford, p. 131-147.

BODU P. 2000 - Que sont devenus les Magdaléniens du Bassin parisien? Quelques éléments de réponse sur le gisement azilien du Closeau (Rueil-Malmaison, France). In : Valentin B., Bodu P., Christensen M. (dir.) 2000, L'Europe Centrale et Septentrionale au Tardiglaciaire. Confrontation des modèles régionaux de peuplement. Actes de la Table-ronde internationale de Nemours - 14, 15, 16 Mai 1997. Nemours : APRAIF, 1 vol. , 361 p., p. 315-339, (Mémoire du Musée de Préhistoire d'Ile-de-France $n^{\circ} 7$ ).

BORDES F. et SONNEVILLE-BORDES D. (de) 1979 - L'azilianisation dans la vallée de la Dordogne. Les données de la Gare de Couze (Dordogne) et de l'abri Morin (Gironde).In : Sonneville-Bordes D. (de) (ed.), La fin des Temps glaciaires en Europe., 1979, p. 449-460.

BOUVIER J.-M. 1973 - Nouvelle diagnose stratigraphique du gisement éponyme de La Madeleine, Tursac, (Dordogne). Compte-rendu de l'Académie des Sciences de Paris, t. 277, Paris, p. 2625-2628.

BOUVIER J.-M. 1982 - Les sols empierrés du Magdalénien IV de la Madeleine (Tursac, Dordogne). In :Combier J. (dir), Les habitats du Paléolithique supérieur. Actes du colloque international en hommage à A. Leroi-Gourhan. Roanne-Villerest, 22-24 juin 1982.p. 194-195. 
BREUIL H. 1912 - Les subdivisions du Paléolithique supérieur et leur signification. Actes du 14ème congrés international d'anthropologie et d'archéologie préhistoriques, Genève, 1912, p. 1-74.

BRÉZILLON M.-N. 1968 - La dénomination des objets en pierre taillée. Matériaux pour un vocabulaire des préhistoriens de langue française.IVème supplément à Gallia Préhistoire, éditions du CNRS.

BUNDGEN B. 2002 - Evolution des comportements techniques au Magdalénien supérieur : les données de l'industrie lithique de La Madeleine (Dordogne), séries récentes.Thèse de Doctorat, Université de Bordeaux I, 1 volume, $323 \mathrm{p}$.

CAPITAN L., PEYRONY D. 1928 - La Madeleine. Son gisement, ses industries, ses œuvres d'art.Publication de l'Institut International d'Anthropologie, $n^{\circ}$ 2, Paris.

CATTELAIN P., PERPÈRE M. 1993 - Tir expérimental de sagaies et de flèches emmanchées de pointes de la Gravette. Archéo-Situla, 17-20, p. 5-28.

CATTELAIN P., PERPÈRE M. 1996 - Tir expérimental de répliques de pointes de la Gravette : bilan et perspectives. Notae Praehistoricae, 16, p. 55-6.

CÉLÉRIER G. 1998 - L'abri-sous-roche de Pont-d'Ambon à Bourdeilles (Dordogne, France). Perspective synthétique. Paleo, $\mathrm{n}^{\circ}$ 10, p. 233-264.

CÉLÉRIER G., MOSS E.-H. 1983 - L'abri sous roche de Pont-d'Ambon à Bourdeilles (Dordogne). Un gisement magdalénien-azilien. Microtraces et analyse fonctionnelle de l'industrie lithique. Gallia Préhistoire, t. 26, 1, p. 81-108.

CÉLÉRIER G., JACQUEMENt P. 2005 - Fragmentation des pointes aziliennes : le site de Pont d'Ambon, Bourdeilles (Dordogne). In : D'un monde à l'autre : les systèmes lithiques pendant le Tardiglaciaire autour de la Méditerranée nord-occidentale. Actes de la table ronde internationale, Aix-enProvence, 6-8 juin 2001.Mémoires de la Société Préhistorique Française, n 40, Paris : SPF, p. 47-56.

CHADELLE J.-P., GENESTE J.-M., PLISSON H. 1991 - Processus fonctionnels de formation des assemblages technologiques dans les sites du Paléolithique supérieur. Les pointes de projectiles lithiques du Solutréen de la grotte de Combe Saunière (Dordogne, France). In : 25 ans d'études technologiques en préhistoire, XIe Rencontres Internationales d'Archéologie et d'Histoire d'Antibes, APDCA, Juans-les-Pins, 1991, p. 275-287.

CHOLLET A., FOUÉRÉ P., HANTAÏ A., LE LICON G. 1999 - L'évolution des choix techniques et économiques entre le Magalénien supérieur et l'Azilien : l'exemple de la grotte du Bois-Ragot (Vienne, France). In Bintz P. et Thévenin A. (dir.), L'Europe des derniers chasseurs. Epipaléolithique et Mésolithique. Actes du Ve congrès UISPP, commission XII, Grenoble (18-23 septembre, 1995).CTHS, Paris, p. 275-286.

CHRISTENSEN M., VALENTIN B. 2004 - Armatures de projectiles et outils. De la production à l'abandon. In : Pigeot N. (dir.), Les derniers Magdaléniens d'Etiolles. Perspectives culturelles et paléohistoriques (l'unité d'habitation Q31), XXXVIIème supplément à Gallia Préhistoire, 2004, Paris : CNRS édition, $\mathrm{p}$.

DEMARS P.-Y., LAURENT P. 1989 - Types d'outils lithiques du Paléolithique supérieur en Europe.Cahiers du Quaternaire $n^{\circ} 14$, Paris : CNRS édition.

FAGNART J.-P., COUDRET P. 2000 - Données récentes sur le Tardiglaciaire du bassin de la Somme.In : G. Pion (dir.), Le Paléolithique Supérieur récent : nouvelles données sur le peuplement et l'environnement.Actes de la Table Ronde de Chambéry, Paris : SPF, p. 113-126, (mémoire XXVIII).

FISCHER A., VEMMING HANSEN P., RASMUSSEN P. 1984 - Macro and micro wear traces on lithic projectile points. Experimental results and prehistoric examples. Journal of Danish Archeology, vol. 3, p. 19-46. 
GENESTE J.-M., PLISSON H. 1990 - Technologie fonctionnelle des pointes à cran solutréennes : l'apport des nouvelles données de la grotte de Combe Saunière (Dordogne). In : J. K. Kozlowski (dir.), Feuilles de pierre : les industries à pointes foliacées du Paléolithique supérieur européen. Liège, ERAUL, 42, p. 293-332.

GENESTE J.-M., PLISSON H. 1993 - Hunting technologies and human behavior : lithic analysis of Solutrean shouldered points. In : H. Knecht, A. Pike-tay, R. White (dir.), Before Lascaux: The Complex Record of the Early Upper Paleolithic. Boca Raton, CRC Press, p. 117-137.

KEELEY L. H. 1981. In : Audouze F., Cahen D., Keeley L. H., Schmider B., Le site magdalénien du Buisson Campin à Verberie (Oise).Gallia Préhistoire, t. 24, fasc. 1, Paris : CNRS édition, p. 99-143.

LADIER E. (dir.) 2003 - Les pointes à cran dans dans les industries lithiques du Paléolithique supérieur récent. Actes de la table ronde de Montauban (26-27 avril 2002). Préhistoire du Sud-Ouest, supplément $\mathrm{n}^{\circ}$ 6, p. 91-99.

LARTET E., CHRISTY H. 1875 - Reliquiae Aquitanicae : being contributions to the Archaeology and Paleontology of Perigord and adjoining provinces of Southern France, 1864-1875.Londres, Rupert Jones Editions.

LEESCH D., CATTIN M.-I., MÜLLER W., 2004 - Hauterive-Champréveyres et Neuchâtel-Monruz. Témoins d'implantations magdaléniennes et aziliennes sur la rive nord du lac de Neuchâtel.Neuchâtel, Service et Musée cantonal d'archéologie (Archéologie neuchâteloise, 31), 237 p.

LENOIR M. 1975 - Observations sur les pointes à crans magdaléniennes dans les gisements de l'Abri Faustin, commune de Cessac (Gironde) et de la Pique, commune de Daignac (Gironde). Bulletin de la Société Préhistorique Française, t. 72, CRSM, nº 4, p. 107-112.

LENOIR M. 1989 - Le Magdalénien en Gironde. In Rigaud J.-P., Organ M., Otte M. Ed. 1989 - Le Magdalénien en Europe " la structuration du Magdalénien ».Actes du colloque de Mayence 1987, XIème congrès UISPP, ERAUL n 38, Liège, 479 p., p. 253-267.

LENOIR M., PAQUEREAU M.-M. 1987 - Aperçu sur le Magdalénien des basses vallées de la Dordogne et de la Garonne. Bulletin de la Société Préhistorique Française, t. 84, n² 2, p. 43-45.

LEROI-GOURHAN A. 1983 - Une tête de sagaie à armatures de lamelles de silex à Pincevent (Seineet-Marne).Bulletin de la Société Préhistorique Française, t. 80, nº 5, p. 154-156.

MOSS E. H., NEWCOMER M. H. 1982 - Reconstruction of tool use at Pincevent : microwear and experiments. Studia Praehistorica Belgica, 2, p. 289-312.

O'FARRELL M. 2004 - Approche fonctionnelle des pointes de la Gravette de Corbiac (Dordogne, France) : fonction du site et modes de chasse. In : Bodu P., Constantin C. (dir.),Approches fonctionnelles en Préhistoire (Nanterre, 24-26 novembre 2000).Paris, SPF (éd.), p. 121-138, (25ème Congrès Préhistorique de France).

O'FARRELL M., PELEGRIN J. 2005 - Les lamelles retouchées ou utilisées de Castanet.In : Le BrunRicalens F., Bordes J.-G., Bon F. (coord.), Productions lamellaires attribuées à l'Aurignacien. Chaînes opératoires et perspectives technoculturelles.Actes du XIVe congrès de l'Union Internationale des Sciences Pré- et Protohistoriques, Université de Liège, 2-8 septembre 2001, p. 103-121.

PELEGRIN J. 2000 - Les techniques de débitage laminaire au Tardiglaciaire : critères de diagnose et quelques réflexions.In : Valentin B., Bodu P., Christensen M. (dir.), L'Europe centrale et septentrionale au Tardiglaciaire, Actes de la table-ronde internationale de Nemours, 13-16 mai 1997. Mémoires du Musée de Préhistoire d'Ile de France, 7, 2000, Nemours : édition APRAIF, p. 73-86.

PIEL-DESRUISSEAUX J.-L. 1998 - Outils préhistoriques. Formes, fabrication, utilisation.Masson, Paris, 3ème édition, $311 \mathrm{p}$. 
PION G. (dir.) 1990 - L’abri de la Fru à Saint-Christophe (Savoie). Gallia Préhistoire, t. 32, CNRS, p. 65-123.

PION G. 1997 - L'abri de la Fru à Saint-Christophe-la-Grotte (Savoie) : l'Azilien ancien du début de l'Alleröd. BSPF, t. 94, n³, p. 315-318.

PLISSON H., GENESTE J.-M. 1989 - Analyse technologique des pointes à cran solutréennes du Placard (Charente), du Fourneau du Diable, du Pech de la Boissière et de Combe Saunière (Dordogne). Paléo, 1, p. 65-109.

PLISSON H. 2005 - Examen tracéologique des pointes aziliennes du Bois-Ragot.In : Chollet A. et Dujardin V. (coord), La Grotte du Bois-Ragot à Gouex (Vienne), Magdaléniens et Aziliens, Essais sur les hommes et leur environnement.Mémoire XXXVIII de la Société Préhistorique Française, Paris, éditions de La Société Préhistorique Française, p. 183-188.

SCHMIDER B. 1988 - Les industries du centre du Bassin parisien au Paléolithique récent : essai de chronologie. In : Otte M (ed.), De la Loire à l'Oder. Les civilisations du Paléolithique final dans le nordouest européen, Actes du colloque de Liège, décembre 1985. Oxford (UK) : BAR, 1988- 2 vol. , 781 p. (ERAUL 25 / British Archeological Reports - International Series 444.), p. 1-13.

SÉRONIE-VIVIEN M., SÉRONIE-VIVIEN M.-R. 1987 - Les silex du Mésozoïque nord-aquitain. Approche géologique de l'étude des silex pour servir à la recherche préhistorique. Supplément au Bulletin de la Société Linéenne de Bordeaux, XV, 135 p.

SIMONNET R. 1999 - De la géographie à la préhistoire : le silex des prépyrénées, résultats et réflexions sur les perspectives et les limites de l'étude des matières premières lithiques. Paléo, $\mathrm{n}^{\circ} 11$, p. $71-88$.

SIMONNET R. (à paraître, en collaboration avec M.-R. Séronien-Vivien, J. Airvaux, J.-G. Bordes, F. Bon et J. Primault) - Le silex dit « Grain de mil », état de la question.

SONNEVILLE-BORDES D. (de) 1960 - Le Paléolithique supérieur en Périgord.Bordeaux, Edition Delmas, $2 \mathrm{t}$.

SONNEVILLE-BORDES D. (de) 1979 - L'abri de Villepin, site de référence. In : Sonneville-Bordes D. de (ed.) 1979, La fin des Temps glaciaires en Europe. Chronostratigraphie et écologie des cultures du Paléolithique final.Actes du Colloque international de Talence (24-28 mai 1977), colloque international du CNRS n² 271, Paris, édition du CNRS.

SONNEVILLE-BORDES D. (de), PERROT J. 1956 - Lexique typologique du Paléolithique supérieur. Outillage lithique (suite et fin) - V outillage à bord abattu - VI pièces tronquées - VII lames retouchées - VIII pièces variées - IX outillage lamellaire, pointe azilienne. Bulletin de la Société Préhistorique Française, t. 53, nº 9, p. 547.

SORIANO S. 1998 - Les microgravettes du Périgordien de Rabier à Lanquais (Dordogne). Gallia Préhistoire, 40, p. 75-94.

TAYLOR A. 2009 - Diversité des pièces à dos de La Madeleine et Laugerie-Haute Est. Caractérisation et essai d'interprétation.Thèse de Doctorat de l'Université de Paris Ouest-Nanterre-La Défense, 2 volumes, 485 et $85 \mathrm{p}$.

TIXIER J. 1963 - Typologie de l'Epipaléolithique du Maghreb. Mémoire du C.R.A.P.E, II, 212 p.

TURQ A. 2005 - Réflexions méthodologiques sur les études de matières premières lithiques. 1 Des lithothèques au matériel archéologique. Paleo, 17, p. 111-131. 
VALENTIN B. 1995 - Les groupes humains et leurs traditions au Tardiglaciaire dans le Bassin Parisien. Apports de la technologie lithique comparée. Paris : Université de Paris I, Panthéon Sorbonne, 1995. 3 vol. Thèse N.D.

VALENTIN B. 2000 - L'usage des percuteurs en pierre tendre pour le débitage des lames.

Circonstances de quelques innovations au cours du Tardiglaciaire dans le Bassin parisien.In : G. Pion (dir.), Le Paléolithique supérieur récent : nouvelles données sur le peuplement et l'environnement, Actes de la Table Ronde de Chambéry, mars 1999. Mémoires de la Société Préhistorique Française, 28, Paris, SPF ed., p. 253-260.

VALENTIN B. 2005 - La fabrication des armatures et outils en silex des couches aziliennes 3 et 4.In : Chollet A. et Dujardin V. (coord), La Grottte du Bois-Ragot à Gouex (Vienne), Magdaléniens et Aziliens, Essais sur les hommes et leur environnement.Mémoire XXXVIII de la Société Préhistorique Française, Paris, éd. de La Société Préhistorique Française, p. 89-182.

\section{ANNEXES}

Planche 1 - Nucléus à lamelles du niveau 27, Magdalénien IV : $n^{\circ} 1$ et 3 , progression frontale unipolaire $; n^{\circ} 2$, progression frontale décalée à partir de deux plans de frappe hiérarchisés (dessins A. Taylor).
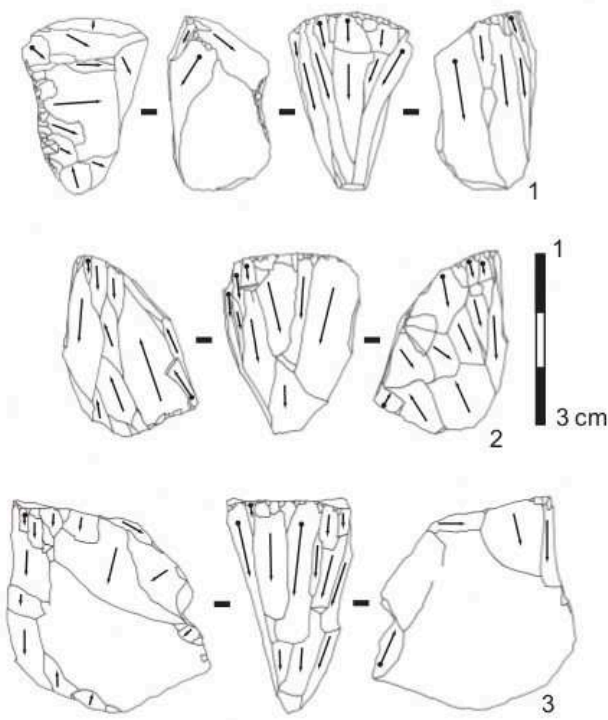

$$
\begin{aligned}
& \text { présence du contrebulbe } \\
& \text { absence du contrebulbe }
\end{aligned}
$$


Planche 2 - Nucléus à lamelles du niveau 25 , Magdalénien $V: \mathrm{n}^{\circ} 1$, progression frontale à partir de deux plans de frappe hiérarchisés; $n^{\circ} 2$ et 3 , progression frontale unipolaire (dessins $A$. Taylor).
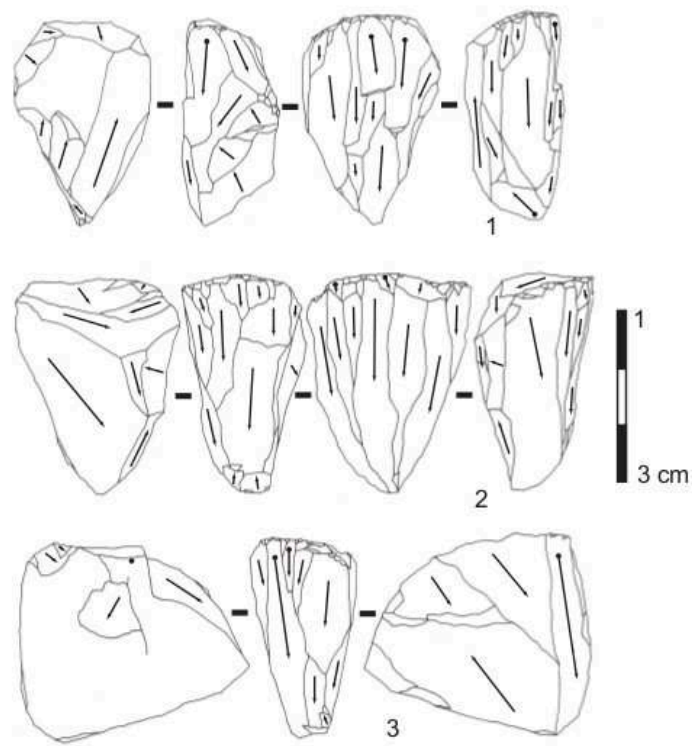

présence du contrebulbe

absence du contrebulbe

Planche 3 - Nucléus à lamelles du niveau 19, Magdalénien VI : progression frontale unipolaire (dessins A. Taylor).
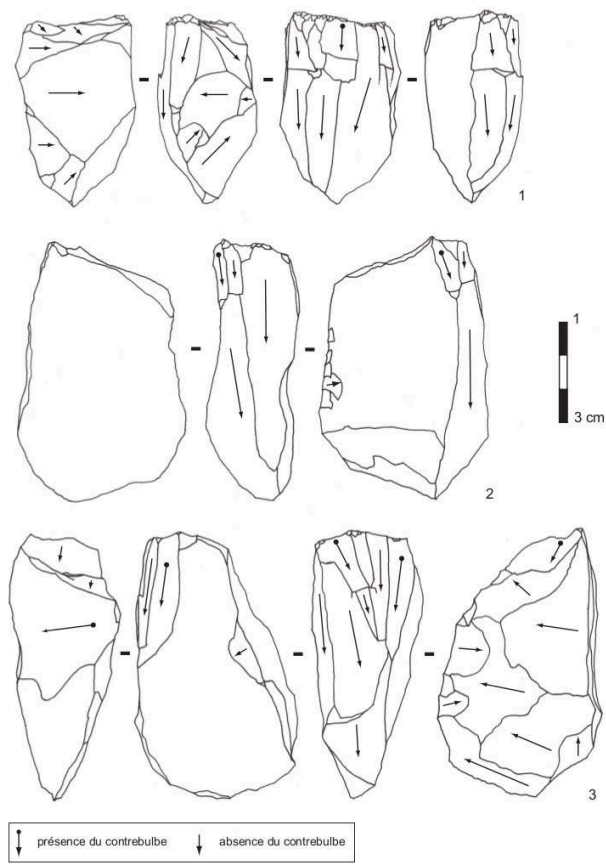
Planche 4 - Lamelles à dos simples : $n^{\circ} 1,2,13$ niveau $28 ; n^{\circ} 3,9$ niveau $26 ; n^{\circ} 4,5,12$ niveau 25 ; $n^{\circ}$ 6-8 niveau $24 ; n^{\circ} 10,11$ niveau 19 (dessins $A$. Taylor).

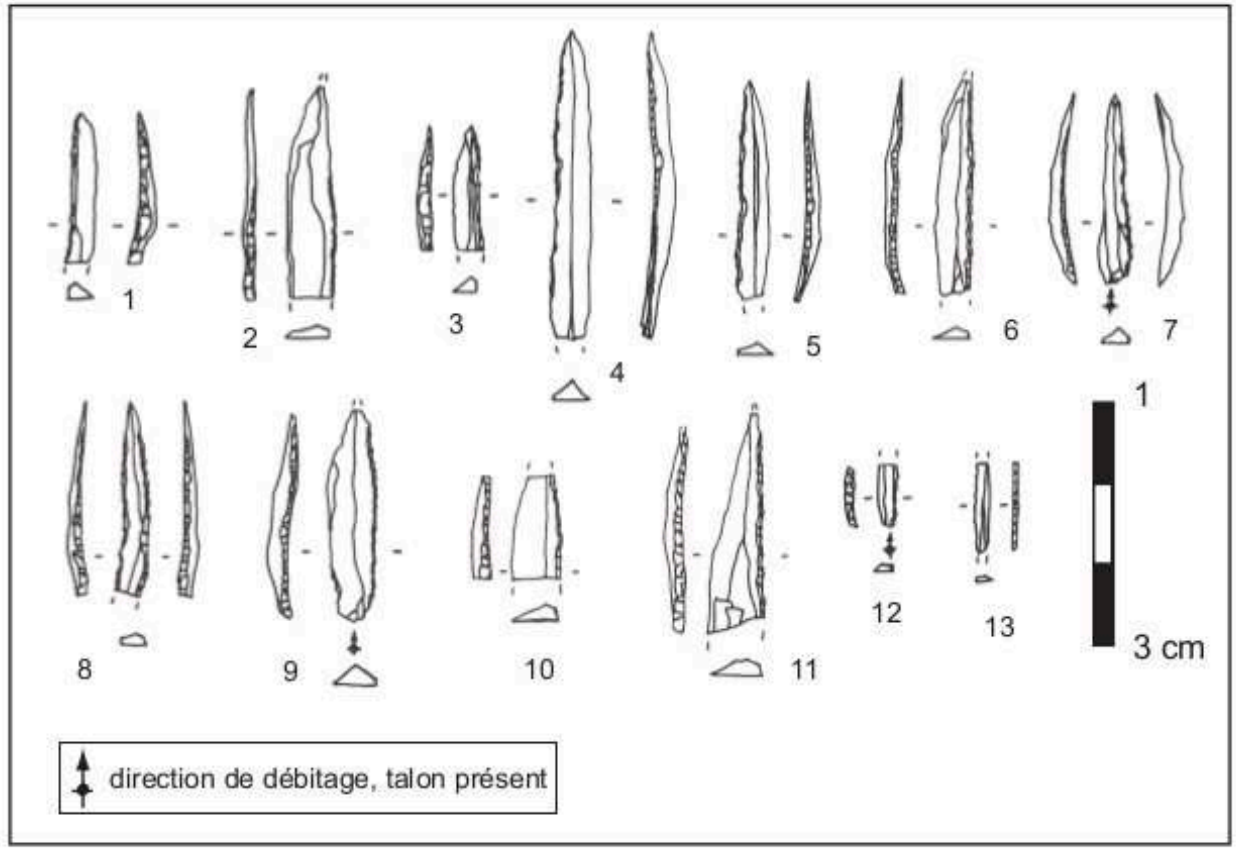

Planche 5 - Pointes lithiques, séries Peyrony. Couche $B$, Magdalénien $V: n^{\circ} 1-4$, pointes à cran magdaléniennes, $n^{\circ} 3$ ébauche ; $n^{\circ} 5-8$, pointes à dos arqués (dessins $A$. Taylor).

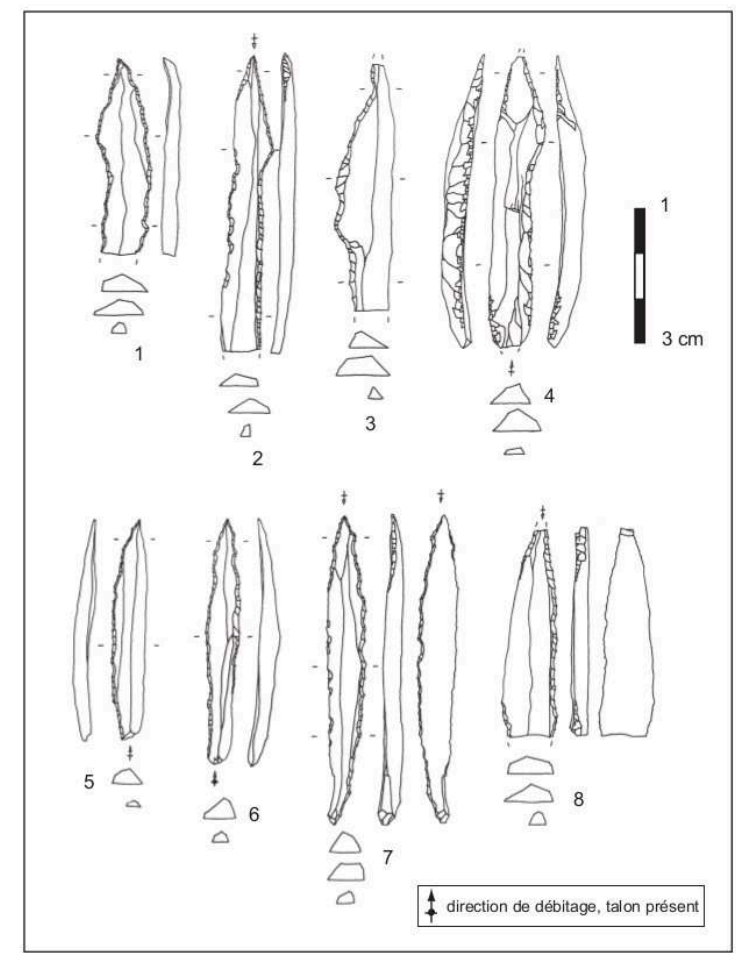


Planche 6 - Pointes lithiques, séries Peyrony. Couche $C$, Magdalénien VI : $n^{\circ} 1-3,5-11$, pointes à cran magdaléniennes ; $n^{\circ} 4$, " pièces à cran » indéterminée ; $n^{\circ} 12$ pointe à dos arqué $; n^{\circ} 13-14$, pointes à dos courbe (dessins $\mathrm{A}$. Taylor).

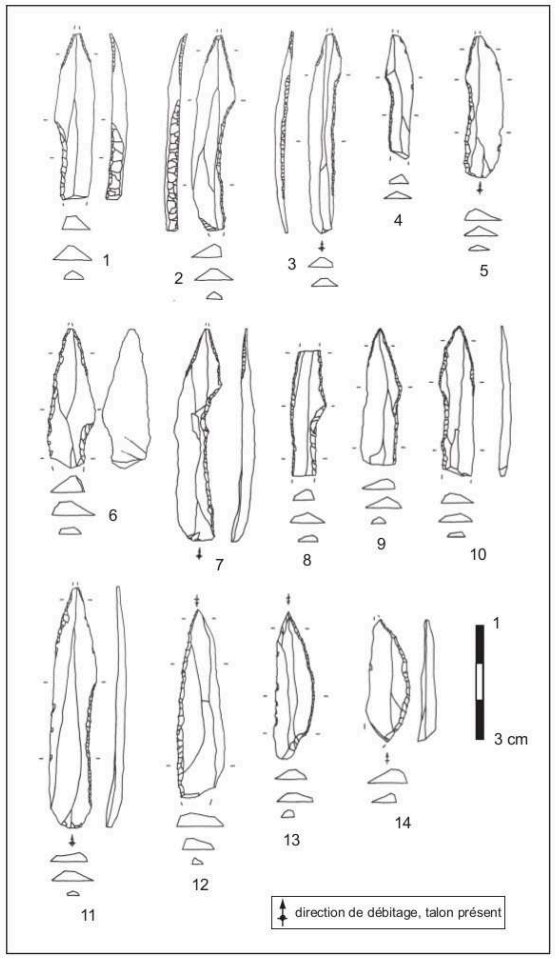

Planche 7 - Lamelles à dos à retouche opposée distale : $n^{\circ} 1-4, n^{\circ} 6$ niveau $25 ; n^{\circ} 5$ niveau $24 ; n^{\circ} 7$ niveau $21 ; n^{\circ} 8$ niveau $26 ; n^{\circ} 9$ niveau $22 ; n^{\circ} 10$ niveau $28 ; n^{\circ} 11$ niveau 16 (dessins A. Taylor).

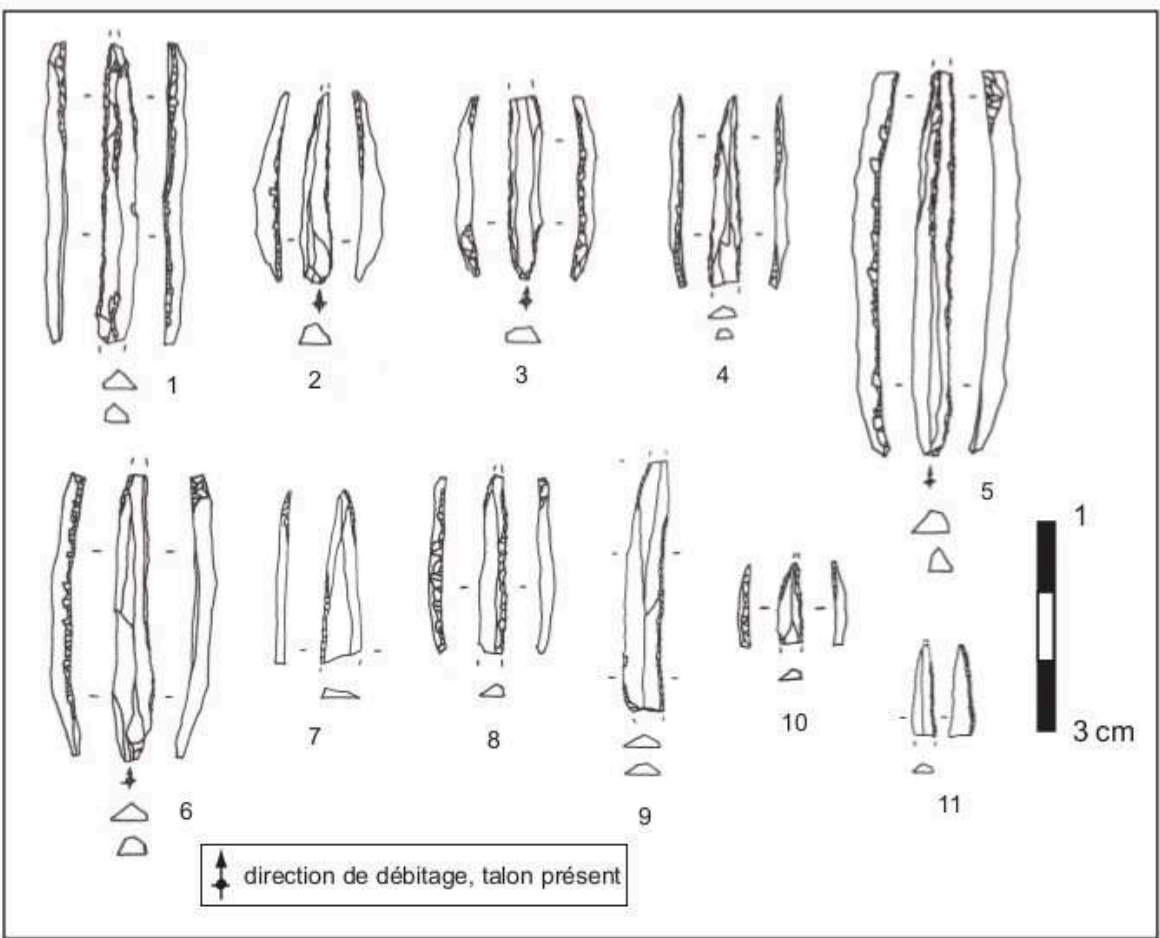


Planche 8 - Lamelles à dos à retouche opposée : $\mathrm{n}^{\circ} 1-3$ niveau $24 ; \mathrm{n}^{\circ} 4,5$ niveau $23 ; \mathrm{n}^{\circ} 6$ niveau 21 ; $\mathrm{n}^{\circ} 7-9$ niveau $22 ; \mathrm{n}^{\circ} 10$ niveau $20 ; n^{\circ} 11-17$ niveau $19 ; \mathrm{n}^{\circ} 18$ niveau 17 (dessins $A$. Taylor).

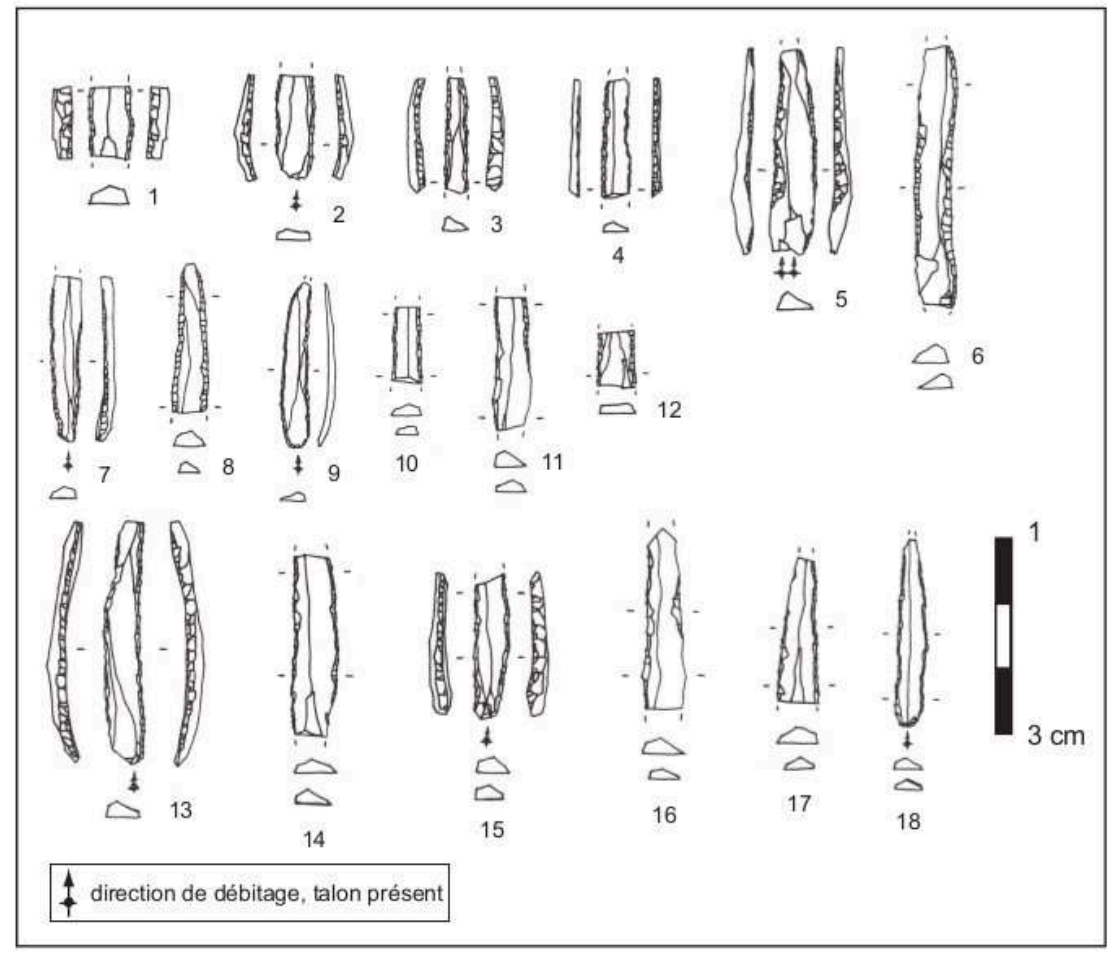

Planche 9 - Lamelles à dos à retouche opposée proximale : $n^{\circ} 1-3$ niveau $28 ; n^{\circ} 4-6$ niveau $27 ; n^{\circ}$ 7,8 niveau $26 ; n^{\circ} 9-11$ niveau $25 ; n^{\circ} 12-14$ niveau $23 ; n^{\circ} 15$ niveau 19 (dessins $A$. Taylor).

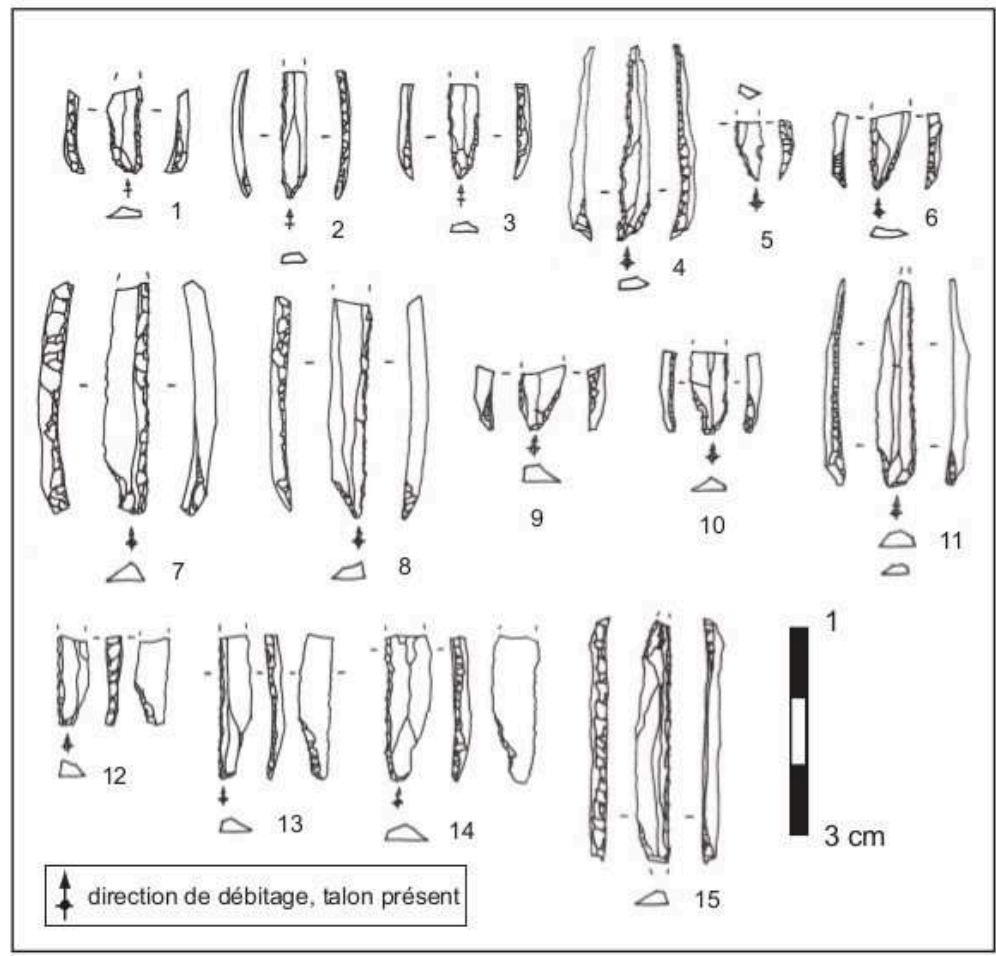


Planche 10 - Quelques exemples d'ébréchures et de fractures complexes diagnostiques d'une utilisation comme éléments de projectile des lamelles à dos.

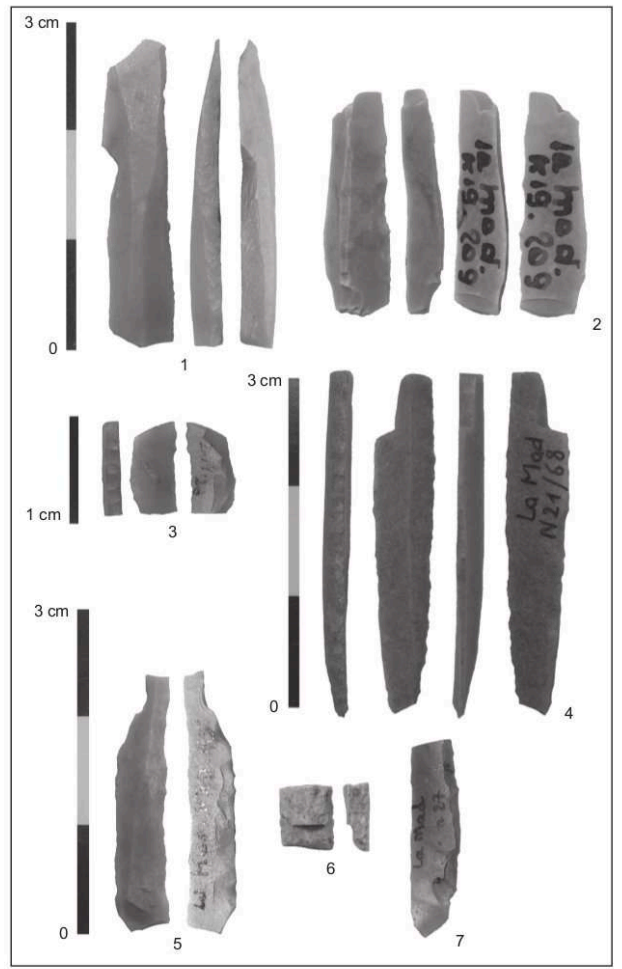

Planche 11 - D'autres exemples d'ébréchures et de fractures complexes diagnostiques d'une utilisation comme éléments de projectiles des lamelles à dos.

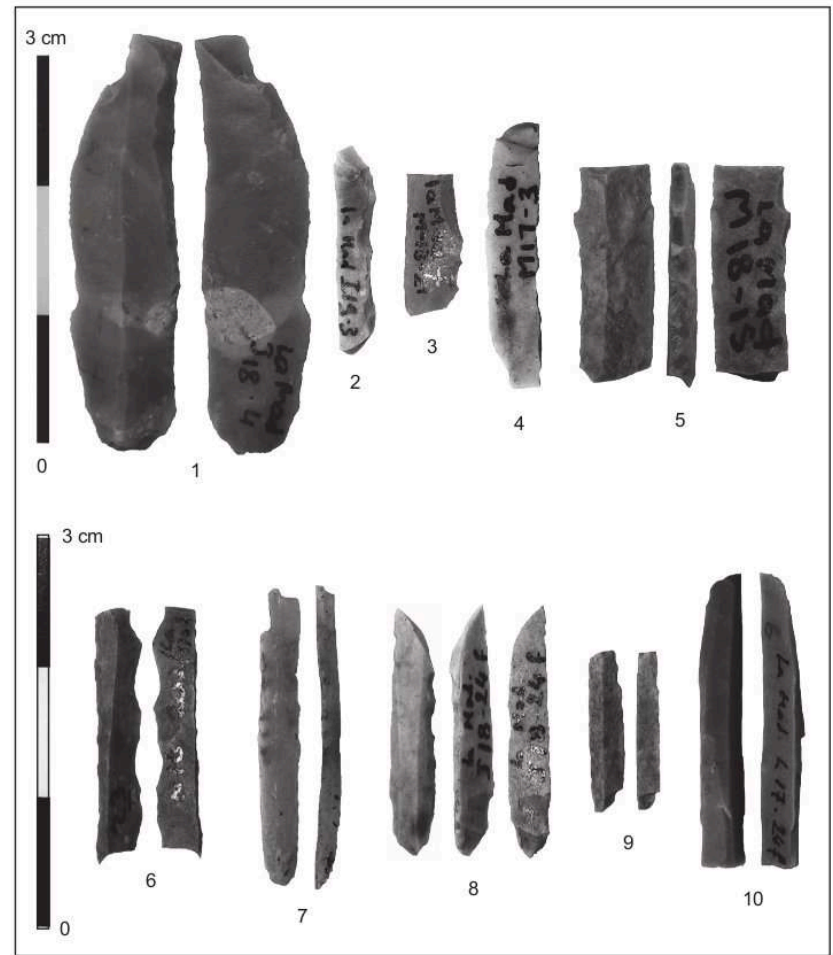


Planche 12 - D'autres exemples d'ébréchures et de fractures complexes diagnostiques d'une utilisation comme éléments de projectiles des lamelles à dos.

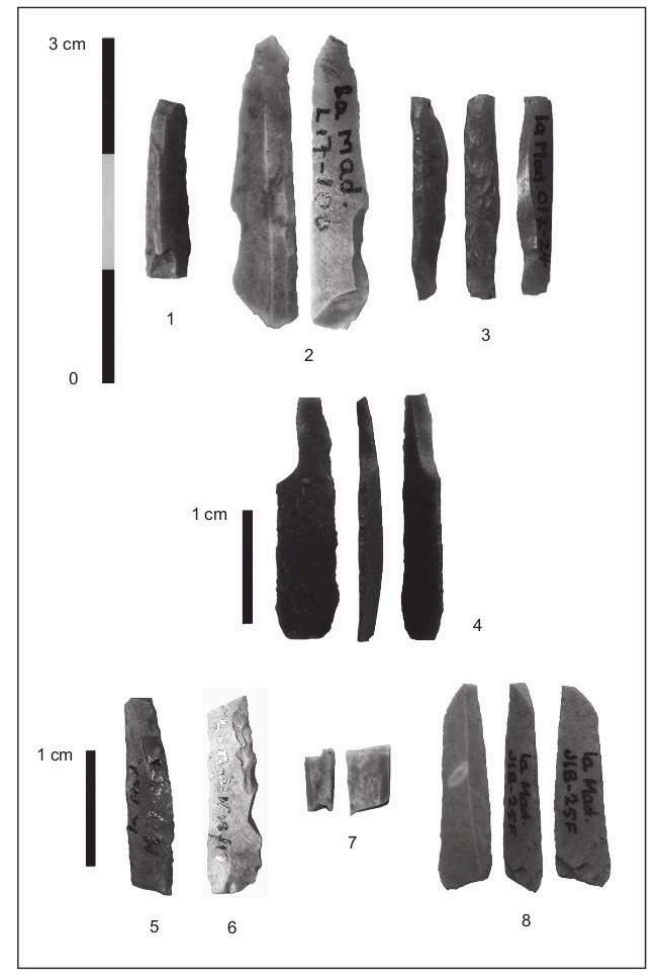

\section{RÉSUMÉS}

La révision technologique et morphologique des lamelles à dos et des pointes lithiques des séries Bouvier et Peyrony de La Madeleine nous a conduit à nous interroger sur le statut et le rôle de ces éléments particuliers au sein de l'équipement de chasse des derniers Magdaléniens.

Tout d'abord du point de vue technologique, la conception des pièces à dos se caractérise par une grande souplesse dans la mise en œuvre des schémas de production : les modalités de débitage et de transformation des supports des pièces à dos sont variées mais répondent à des critères rigoureux quant à leurs objectifs.

Le débitage lamellaire de La Madeleine s'oriente clairement vers la production de supports effilés, une retouche complémentaire (bord opposé au dos ou parties proximales et/ou distales) intervenant si nécessaire comme solution technique de rectification de la silhouette de l'objet. Dans certains cas, cette retouche complémentaire modifiant significativement la morphologie du support pourrait intervenir dans la confection de classes particulières de lamelles à dos. Ces différentes modalités de retouche complémentaire se développent notamment à partir du Magdalénien V et VI, parallèlement à l'apparition de nouvelles têtes de projectiles lithiques (pointes à dos, pointes à cran) et osseuses soulignant l'évolution de la conception de l'armement. Dans ce cadre évolutif, le renforcement des critères morpho-technologiques des lamelles à dos pourrait traduire la redéfinition voire la spécialisation de leur statut.

The technological and morphological revision of the backed bladelets and lithic points from the Peyrony and Bouvier series, brought us to question the status and role of these particular objects in the hunting equipment of the last Magdalenians. A great flexibility in the production schemes characterizes the conception of backed bladelets, the methods of debitage and transformation of 
blanks into backed pieces varying but following the same strict criteria as to their aim. Bladelet debitage at La Madeleine clearly aims at the production of tapered blanks, a complementary retouch adjusting the outline of the objects if necessary (edge, proximal or distal extremities). In some cases, this complementary retouch clearly modifies the cutting edge features and could then intervene in the making of particular types of backed bladelets. The schemes of retouch of these objects evolve progressively but clearly during the occupation of the shelter. The evolution of the lithic insets retouch schemes is clearly more effective during the Magdalenian V and VI, when curved-backed points first, then shouldered points are made, and the harpoon's technology develops in antler working, emphasizing the evolution in the conception of hunting equipment. This evolution could be the result of a change in status of previous conceptions of projectile points, which no longer represent the only technical options. The greater care taken in the shaping of some bladelets could come within the intent of better defining the role of hunting equipment existing before the introduction of new projectile points. Their evolution could then be part of a global modification of magdalenian hunting equipment and capture techniques.

\section{INDEX}

Mots-clés : La Madeleine, Sud-Ouest, France, Magdalénien supérieur, industrie lithique, armatures, pièces à dos, pointes lithiques, technologie, morphologie

Keywords : La Madeleine, Southwestern France, Upper Magdalenian, lithic industry, projectile tips, backed pieces, lithic insets, lithic points, technology, morphology

\section{AUTEUR}

\section{ALEXIS TAYLOR}

Université Paris X - Nanterre UMR 7055 Préhistoire et Technologie, MAE, 21, Allée de

l'Université, 92000 Nanterre, France - alexis.taylor@wanadoo.fr 